DEVIATION OF LONG-PERIOD TIDES FROM EQUILIBRIUM: KINEMATICS AND GEOSTROPHY

Gary D. Egbert and Richard D. Ray

Printed in the United States of America Reprinted from Journal of Physical Oceanography Vol. 33, No. 4, April 2003

○ 2003 American Meteorological Society 


\title{
Deviation of Long-Period Tides from Equilibrium: Kinematics and Geostrophy
}

\author{
GARY D. EGBERT \\ College of Oceanic and Atmospheric Sciences, Oregon State University, Corvallis, Oregon \\ RICHARD D. RAY \\ NASA Goddard Space Flight Center, Greenbelt, Maryland
}

(Manuscript received 10 August 2001, in final form 4 October 2002)

\begin{abstract}
New empirical estimates of the long-period fortnightly (Mf) tide obtained from TOPEX/Poseidon (T/P) altimeter data confirm significant basin-scale deviations from equilibrium. Elevations in the low-latitude Pacific have reduced amplitude and lag those in the Atlantic by $30^{\circ}$ or more. These interbasin amplitude and phase variations are robust features that are reproduced by numerical solutions of the shallow-water equations, even for a constant-depth ocean with schematic interconnected rectangular basins. A simplified analytical model for cooscillating connected basins also reproduces the principal features observed in the empirical solutions. This simple model is largely kinematic. Zonally averaged elevations within a simple closed basin would be nearly in equilibrium with the gravitational potential, except for a constant offset required to conserve mass. With connected basins these offsets are mostly eliminated by interbasin mass flux. Because of rotation, this flux occurs mostly in a narrow boundary layer across the mouth and at the western edge of each basin, and geostrophic balance in this zone supports small residual offsets (and phase shifts) between basins. The simple model predicts that this effect should decrease roughly linearly with frequency, a result that is confirmed by numerical modeling and empirical $\mathrm{T} / \mathrm{P}$ estimates of the monthly $(\mathrm{Mm})$ tidal constituent. This model also explains some aspects of the anomalous nonisostatic response of the ocean to atmospheric pressure forcing at periods of around 5 days.
\end{abstract}

\section{Introduction}

The long-period tides (hereinafter LPT) are generated by the small, zonal components of the gravitational tidal potential. They have periods of 5 days and longer (Table 1). Early investigations of the LPT, comprehensively reviewed by Wunsch (1967), were largely theoretical and focused on the question of how close these lowfrequency oscillations should be to static equilibrium, with elevations purely zonal and proportional to the tidal potential:

$$
\zeta_{\mathrm{EQ}}(\theta)=\alpha\left[1 / 2-(3 / 2) \sin ^{2} \theta\right],
$$

where $\theta$ is latitude. Analytical results for the case of a global ocean of uniform depth showed that zonal currents in geostrophic balance with meridional pressure gradients could support significant deviation of elevations from equilibrium. Allowing for friction, ocean basins of complex shape, and variable bottom topography complicates analysis significantly, making it difficult to reach firm theoretical conclusions about the response of the real ocean to the tidal forcing (Wunsch 1967).

Corresponding author address: Dr. Gary D. Egbert. College of Oceanic and Atmospheric Sciences, Ocean Admin. Building 104, Oregon State University, Corvallis, OR 97331-5503.

E-mail: egbert@coas.oregonstate.edu
Until fairly recently observational data have also failed to provide clear constraints on the nature of the LPT response. Even the largest LPT constituent (Mf) has an amplitude of only a few centimeters, so long time series are required to separate the periodic tidal signal from other energetic low-frequency oceanographic variations. Although analysis of Pacific Ocean tide-gauge data by Wunsch (1967) and Luther (1980), as well as altimeter studies by Cartwright and Ray (1990a), Ray and Cartwright (1994), and Desai and Wahr (1995) clearly show that Mf is not in equilibrium with the tidal potential, the exact nature of the global nonequilibrium response is still debated (Miller et al. 1993; Wunsch et al. 1997).

Based on an analysis of available island tide gauge records, Wunsch (1967) concluded that Mf elevations deviated significantly from equilibrium in the Pacific. He suggested that these deviations could be explained in terms of quasigeostrophic dynamics, with the oceanic response dominated by small-scale, nearly resonant, barotropic Rossby waves. Later analysis of a more extensive set of tide gauge records from the Pacific by Luther (1980) showed that the deviation of Mf from equilibrium was essentially in phase across the basin, a spatial pattern not particularly suggestive of Rossby waves. Miller et al. (1993) extended this study with a 
TABLE 1. Major long-period equilibrium tidal constituents.

\begin{tabular}{clcrc}
\hline \hline $\begin{array}{c}\text { Doodson } \\
\text { No. }\end{array}$ & Name & $\begin{array}{c}\text { Speed } \\
\left({ }^{\circ} \mathrm{h}^{-1}\right)\end{array}$ & $\begin{array}{c}\text { Period } \\
\text { (days) }\end{array}$ & $\begin{array}{c}\text { Amplitude } \alpha \\
(\mathrm{mm})\end{array}$ \\
\hline 055.565 & & 0.00221 & 6798.37 & 12.21 \\
056.554 & Sa & 0.04107 & 365.26 & 2.15 \\
057.555 & Ssa & 0.08214 & 182.62 & 13.54 \\
063.655 & MSm & 0.47152 & 31.81 & 2.94 \\
065.455 & Mm & 0.54438 & 27.55 & 15.38 \\
073.555 & MSf & 1.01590 & 14.77 & 2.55 \\
075.555 & Mf & 1.09803 & 13.66 & 29.12 \\
083.655 & MSt & 1.56955 & 9.56 & 1.06 \\
085.455 & Mt & 1.64241 & 9.13 & 5.58 \\
093.555 & $\mathrm{MSq}$ & 2.11393 & 7.10 & 0.89 \\
095.355 & $\mathrm{Mq}$ & 2.18678 & 6.86 & 0.74 \\
$0 \times 3.455$ & & 2.65830 & 5.64 & 0.22 \\
\hline
\end{tabular}

Except for the 18.6-yr line (055.565), nodal and perigean modulation lines are not listed. In shallow water, observed $\mathrm{Mm}$ and $\mathrm{Mf}$, and especially MSf, can be enhanced by nonlinear interactions between short-period tides. Observed $\mathrm{Sa}$ and $\mathrm{Ssa}$ are primarily meteorological, not gravitational. Amplitudes include body-tide reduction factor $\gamma_{2}$ $=0.693$.

combination of additional island tide gauge analyses and numerical modeling, and they concluded that the nonequilibrium LPT response was dominantly basin scale, with a $10^{\circ}-40^{\circ}$ phase lag of the low-latitude Pacific relative to the Atlantic. Modeling studies by Kagan et al. (1976), Carton (1983), and Wunsch et al. (1997) confirm this basin-scale pattern, although none of these authors emphasized or attempted to explain this largescale feature. Miller et al. (1993) argued that the Pacific basin phase lag could be explained best in terms of gravity waves propagating from the Arctic, through the Atlantic and Indian Oceans, and finally into the Pacific. In this picture quasigeostrophic dynamics are of limited importance in determining the large-scale nonequilibrium response.

Altimeter data have now allowed direct mapping of elevations of short-period tidal constituents in the open ocean with an accuracy of $1-2 \mathrm{~cm}$ (e.g., Shum et al. 1997). The same techniques can obviously be applied to the LPT, but this is a challenge since amplitudes (Table 1) are not much greater than the presently achievable uncertainties. Early efforts with geodetic satellite (GEOSAT) data (Cartwright and Ray 1990a; Ray and Cartwright 1994) were limited to zonally averaged estimates of the largest constituent Mf. These results confirmed the deviation of Mf from equilibrium, but did not resolve interbasin differences. Desai and Wahr (1995) presented the first purely empirical maps of LPT constituents, based on analysis of two years of TOPEX/ Poseidon (T/P) data. Although these initial maps were noisy, they nonetheless show the basin-scale variations of amplitude and phase emphasized by Miller et al. (1993). With the availability now of over $8 \mathrm{yr}$ of T/P altimeter data, more definitive empirical maps of the LPT are possible.

In this paper we present a new empirical map of Mf, confirming that there are significant basin-scale deviations from equilibrium. We then show that this largescale pattern can be explained with a very simple model of interconnected basins. The model is largely kinematic: self-consistent (i.e., mass conserving) equilibrium tides within each basin would result in offsets at basin boundaries. These offsets drive mass flux between basins, predominantly in a narrow boundary layer that extends across the mouth and then along the western edge of each basin. Geostrophic balance of the currents in this layer support small residual offsets between basins, allowing basin-to-basin variations in LPT elevations.

The paper is organized as follows. In section 2 we present the new empirical Mf estimates and show that the observed large-scale deviation from equilibrium is reproduced by numerical models with both realistic and highly schematic bathymetry and basin geometries. To illuminate the mechanisms responsible for the largescale nonequilibrium response, we focus on the case of a schematic (constant 4000 -m depth, rectangular basins) global ocean. The dynamics in this simplified geometry is examined in detail in section 3, where we show that a very simple, almost purely kinematic, model reproduces the main features in the empirical T/P estimates and numerical solutions. In section 4 some variants and extensions are considered, including the frequency dependence of the nonequilibrium LPT and the effects of friction. We also briefly consider the implications of these results for nontidal atmospherically forced barotropic motions.

\section{LPT elevations from TOPEX/Poseidon and from models}

Figures $1 \mathrm{a}$ and $1 \mathrm{~b}$ show empirical estimates of the amplitudes and phase lags of the Mf tide deduced from T/P altimeter data. The data spanned the interval September 1992-June 2000. An empirical, binning-type method was used, a slight variation on the method employed by Cartwright and Ray (1990b) and by Desai and Wahr (1995). The usual altimeter corrections (for media delay, etc.) were applied to the height measurements, including a correction for solid body tides and for short-period ocean tides (Ray 1999). All data falling within bins of a certain size were then subjected to tidal analysis for the constituents Sa, Ssa, Mm, MSf, Mf, and Mt. The primarily meteorological tides, Sa and Ssa, have been deduced from T/P data by others (e.g., Nerem et al. 1994) and are not of interest here, while the smaller lines, MSf and Mt, are not expected to be sensible with present noise levels, but were included for variance reduction and correlation checks. Because the long-period tides are so small relative to both measurement noise and background oceanographic noise and because their large-scale structure is predominantly zonal, we have adopted a rather large bin size, elongated in the zonal direction: $8^{\circ}$ (longitude) $\times 2^{\circ}$ (latitude). The bins were 

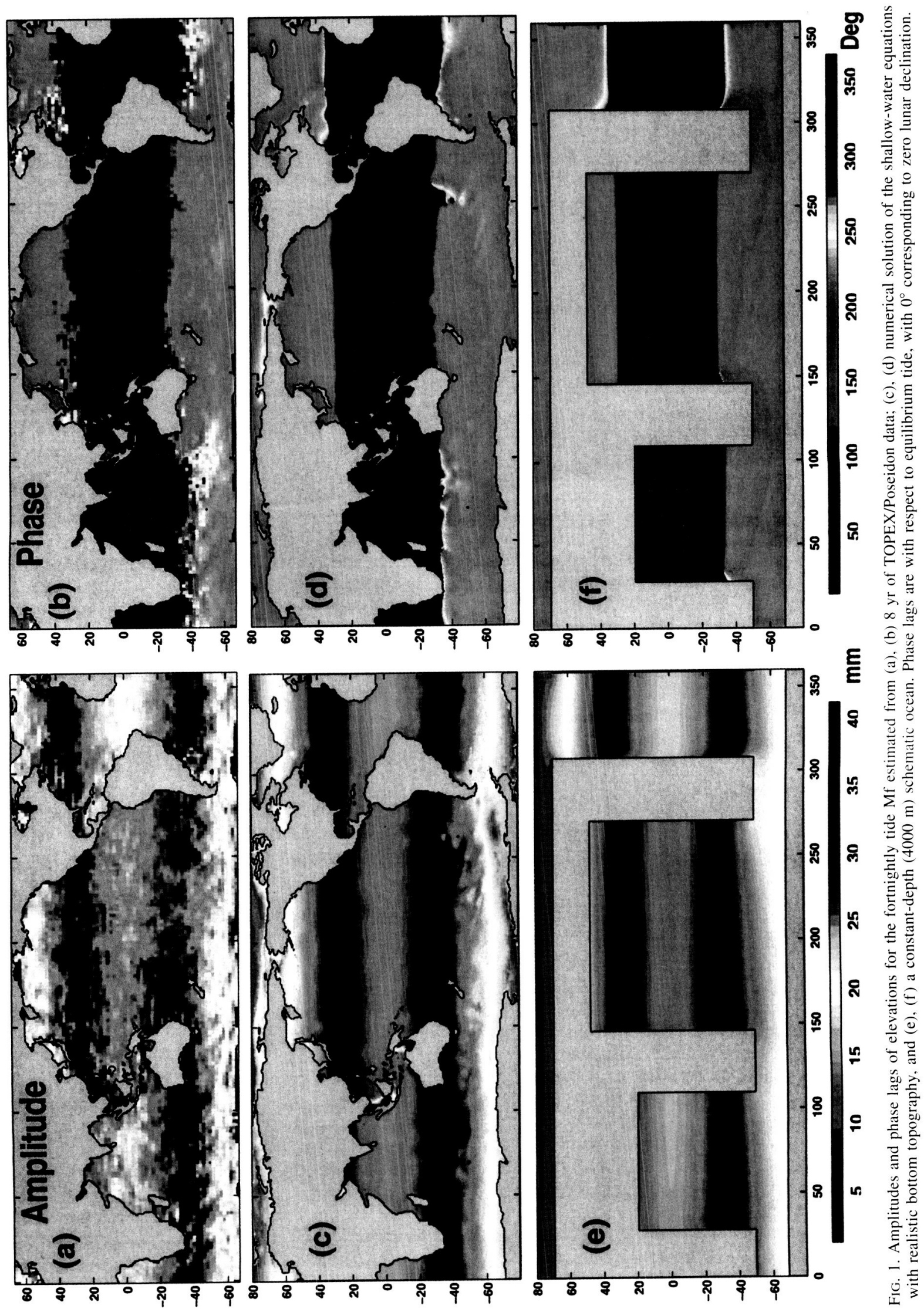
aligned on a $2^{\circ}$ overlapping grid, covering the $\mathrm{T} / \mathrm{P}$ region between latitudes $\pm 66^{\circ}$. Data in each bin were analyzed independently from other bins. Within each bin the standard errors for $\mathrm{Mf}$ are of order $5 \mathrm{~mm}$, increasing to several times that in regions of intense mesoscale variability. The altimetric estimates were corrected for solid-earth loading by applying the iterative procedure described in appendix A of Cartwright and Ray (1991); the results therefore correspond to a bottom-relative tide, as would be measured by a common tide gauge.

The T/P estimates in Fig. 1 exhibit clear basin-scale variations in amplitude and phase. Amplitudes in the Tropics are largest in the Atlantic, smallest in the Pacific, and intermediate in the Indian ocean. The elevations in the Pacific lag those in the Atlantic by $30^{\circ}$ or more, in good agreement with the tide-gauge and modeling results reported by Miller et al. (1993). As with the amplitudes, phase lags in the Indian Ocean are intermediate between those of the other two basins. Within each basin, elevations are dominantly zonal, although some cross-basin amplitude gradients are evident, especially in the Pacific.

Figures $1 \mathrm{c}$ and $1 \mathrm{~d}$ show amplitudes and phases of $\mathrm{Mf}$ elevations from a numerical hydrodynamic model. These results were computed by time stepping the nonlinear shallow water equations, formulated in terms of elevation $\zeta$ and volume transport $U$, on a $1 / 6$ nearly global $\left(80^{\circ} \mathrm{S}-80^{\circ} \mathrm{N}\right)$ grid, with bathymetry $H$ from a combination of the Smith and Sandwell (1997) and ETOPO5 (National Geophysical Data Center 1992) databases. The model was forced with the gravitational potential (adjusted for effects of body tides, and ocean loading and self-attraction) for six tidal constituents $\left(\mathrm{M}_{2}, \mathrm{~S}_{2}, \mathrm{~K}_{1}, \mathrm{O}_{1}, \mathrm{Mf}\right.$, and $\left.\mathrm{Mm}\right)$ for 120 days, and outputs from the final 60 days were harmonically analyzed. Dissipation in the model included a quadratic bottom friction term $\mathcal{F}=c_{D} \mathbf{U} v / H$ (where $v$ is the total water speed, $\mathbf{U}$ are volume transports, and $c_{D}=0.003$ ), and horizontal viscosity with a constant eddy coefficient $A_{h}=$ $10^{3} \mathrm{~m}^{2} \mathrm{~s}^{-1}$. The relatively large value for $A_{h}$ [which is comparable to that used by Wunsch et al. (1997)] is required for numerical stability. (In fact, $A_{h}$ can be set to zero if advection terms are dropped from the shallow water equations; the resulting Mf solutions are very similar to those shown here.) The hydrodynamic solution is generally much smoother than the empirical T/P map, but large-scale patterns are similar. In particular, elevations in the low-latitude Pacific exhibit significant phase lags and are of reduced amplitude. Amplitudes and phases in the Indian Ocean again fall between those for the Atlantic and Pacific.

Both the numerical and empirical solutions contain finer-scale details. For the numerical solution most of these short-wavelength features are clearly the subtle expressions of Rossby waves, in many cases trapped by topography (e.g., note the very distinctive amphidromic phase anomaly in the southeastern Pacific Ocean). Finerscale details in the empirical solution are too contam- inated with noise to allow unambiguous interpretation, but some of these are also suggestive of quasigeostrophic dynamics and topographic effects. Data assimilation solutions for Mf, obtained as in Egbert et al. (1994) and Egbert and Erofeeva (2002) (not shown), match more of the details in the empirical map, and, depending on assumptions about friction and the degree of fit to the $\mathrm{T} / \mathrm{P}$ data, can contain many propagating and topographically trapped Rossby waves. We defer discussion of these assimilation solutions, barotropic currents, and Rossby waves to a future paper. There are also some local amplifications of LPT elevations in the numerical solution, which are not evident in the T/P estimates (e.g., north of Australia). These small-scale features are sensitive to details in the model configuration, and probably should not be taken too seriously. Here we focus on providing a simple dynamical explanation for the much more robust basin-scale deviations of LPT elevations from equilibrium.

We first demonstrate that the basic pattern of interbasin variations is reproduced in a numerical solution for a highly simplified schematic model of the ocean. For the results of Figs. 1e and If the oceans are modeled as three rectangular basins (nominally the Indian, Pacific and Atlantic), all connected at the southern boundary $\left(50^{\circ} \mathrm{S}\right)$ to a zonally connected schematic Southern Ocean. The Atlantic basin is also connected at the top $\left(70^{\circ} \mathrm{N}\right)$ to a simplified Arctic Ocean. Water depth is a constant $4000 \mathrm{~m}$. The shallow-water equations were solved for this simplified geometry by time stepping on a $1 / 2^{\circ}$ grid, in this case forced only by the Mf potential, and with a linear drag law $\mathcal{F}=r \mathbf{U} / H$ with $r=0.03 \mathrm{~m}$ $\mathrm{s}^{-1}$. With the drag coefficient set to this relatively high value the Mf solutions are very simple, with little evidence in the elevations for the barotropic Rossby waves seen in Figs. 1c and 1d. In fact, Rossby waves can and do appear in the schematic ocean model when $r$ is reduced to smaller values, most clearly in the velocity at the western edges of the basins. However, even in this case the basin-scale pattern of elevation variations remains essentially the same.

At the largest scale the elevations for the schematic model are strikingly similar to both the empirical T/P and realistic bathymetry solutions. In all cases elevations are dominantly zonal within each basin, with clear and consistent variations of amplitude and phase between basins. To compare these large-scale differences among the various solutions we consider zonal averages of elevation, computed separately for each basin. These are shown in Fig. 2 for the three solutions of Fig. 1 as real and imaginary parts. The general similarity of the basinwide zonal averages for the three solutions is again apparent, although some more subtle differences are now clearer. Most notably, differences between basins have somewhat larger amplitude for the empirical estimates, and the response in the Indian Ocean is somewhat different for the schematic model (i.e., the imaginary part lies slightly below the corresponding curve 

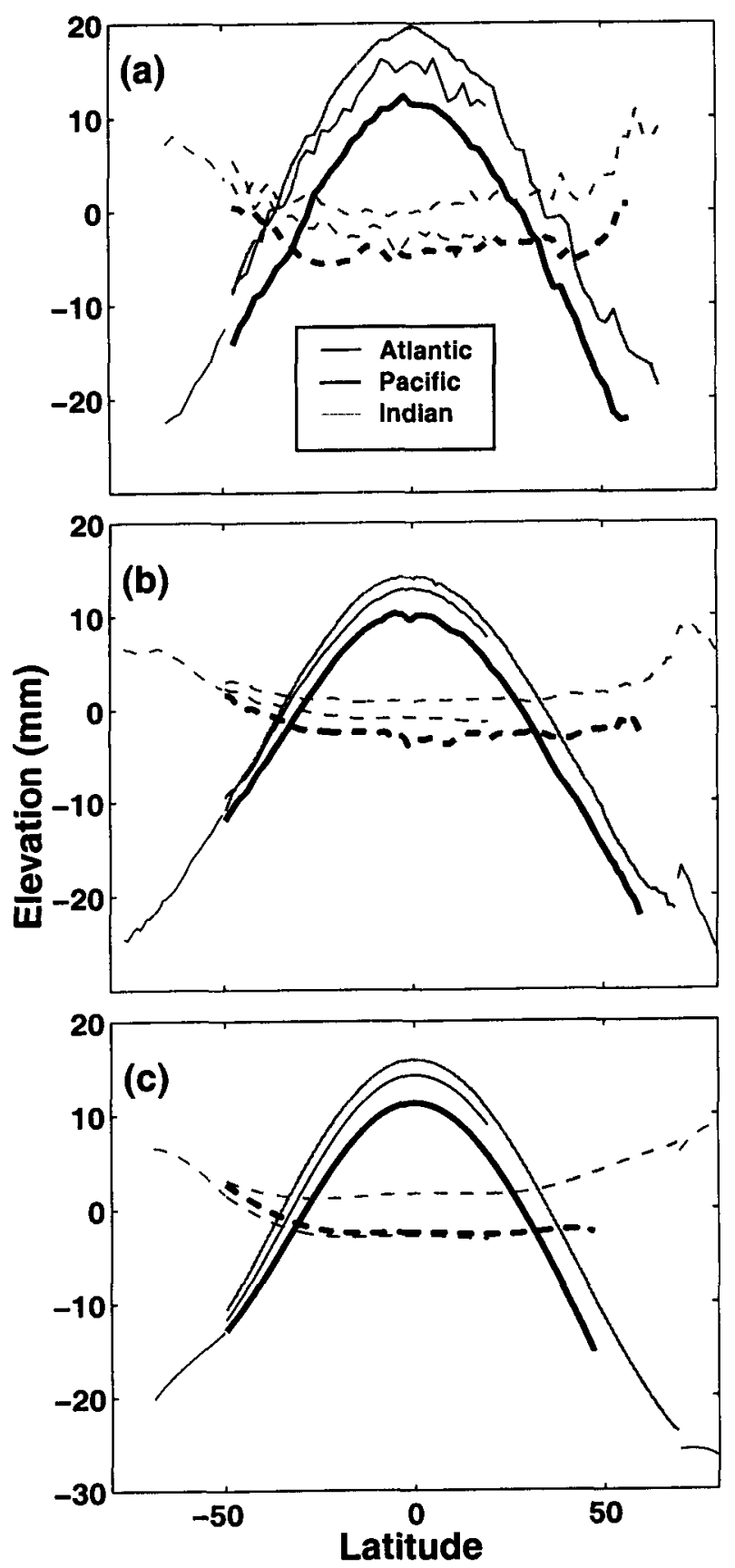

FIG. 2. Basin-by-basin zonal averages of elevations for the three Mf solutions shown in Fig. 1: (a) empirical T/P estimates, (b) numerical solution with realistic ocean basins and bottom topography, and (c) numerical solution for schematic ocean. Real $(A \cos G)$ parts are solid, imaginary $(-A \sin G)$ parts are dashed, where $A$ and $G$ are the amplitudes and phase lags as in Fig. 1. Curves for each basin are distinguished by line thickness; these are also readily identified by noting the different latitude extent for each basin.

for the Pacific). This last difference most probably reflects the fact that the simplified geometry of the schematic ocean ignores connections between the Indian and Pacific Oceans via the Indonesian Throughflow. However, the similarities between the three cases considered

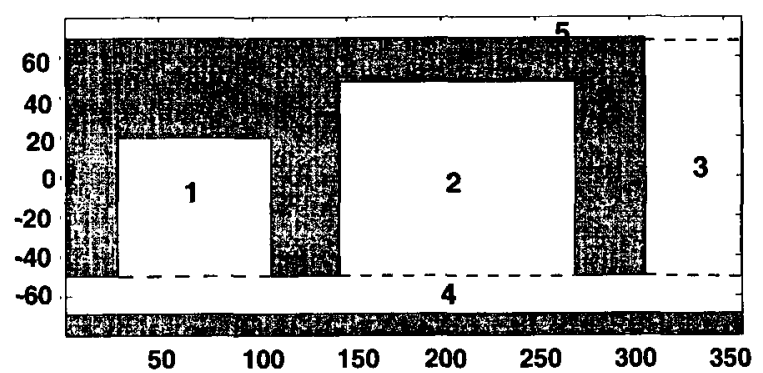

FIG. 3. Division of the schematic ocean into five distinct basins, separated by imaginary barriers.

here are much more striking than the differences, suggesting that the basic pattern of deviation from equilibrium must have a simple explanation in term of the gross geometry of the ocean basins.

\section{An approximate theory for the nonequilibrium LPT}

The large-scale pattern of deviations from equilibrium is captured well by the schematic model (Figs. 1e-f), and so we focus on understanding the dynamics in this simplified geometry where division into individual basins is simple and unambiguous. We consider the frequency-domain shallow-water equations with linear friction:

$$
\begin{aligned}
(\mathbf{F}+i \omega \mathbf{I}) \mathbf{U} & =-c^{2} \boldsymbol{\nabla}\left(\zeta-\zeta_{\mathrm{EQ}}\right) \\
\mathbf{F} & =\left[\begin{array}{cc}
\kappa & -f \\
f & \kappa
\end{array}\right] \quad c^{2}=g H \\
\boldsymbol{\nabla} \cdot \mathbf{U} & =-i \omega \zeta .
\end{aligned}
$$

Here, $\mathbf{U}=(U, V)$ is volume transport, $\zeta$ is elevation, $\kappa=r / H$ is a linear friction coefficient $\left(r=0.03 \mathrm{~m} \mathrm{~s}^{-1}\right.$ unless otherwise noted), $H(=4000 \mathrm{~m})$ is water depth, $\zeta_{\mathrm{EQ}}$ is the equilibrium LPT potential of (1), and $\omega$ $\left(=5.3234 \times 10^{-6} \mathrm{rad} \mathrm{s}^{-1}\right.$ for Mf $)$ is angular frequency. Following Garrett (1975), we imagine barriers dividing the domain into a series of separate basins (Fig. 3), and write the solution in each as the sum of a closed basin solution $\left(\zeta^{0}\right)$ forced by the LPT potential (with zero normal flow at all boundaries) and an unforced solution $\left(\zeta^{v}\right)$ satisfying appropriate inhomogeneous normal flow boundary conditions [with $\zeta_{\mathrm{EQ}} \equiv 0$ in (2)]. The boundary conditions for $\zeta^{V}$ are determined by the requirement that elevations (and volume fluxes) match for adjoining basins, and can be found explicitly by solving a system of coupled integral equations (Garrett 1975). For the zonally averaged basin-scale response to forcing by the zonal LPT potential, these integral equations can be reduced approximately to a very small system of ordinary linear equations, which can be expressed in terms of fundamental solutions computed for each basin separately. We thus first consider (2)-(3) in closed rectangular basins, forced either by the LPT potential $\zeta_{\mathrm{EQ}}$ 

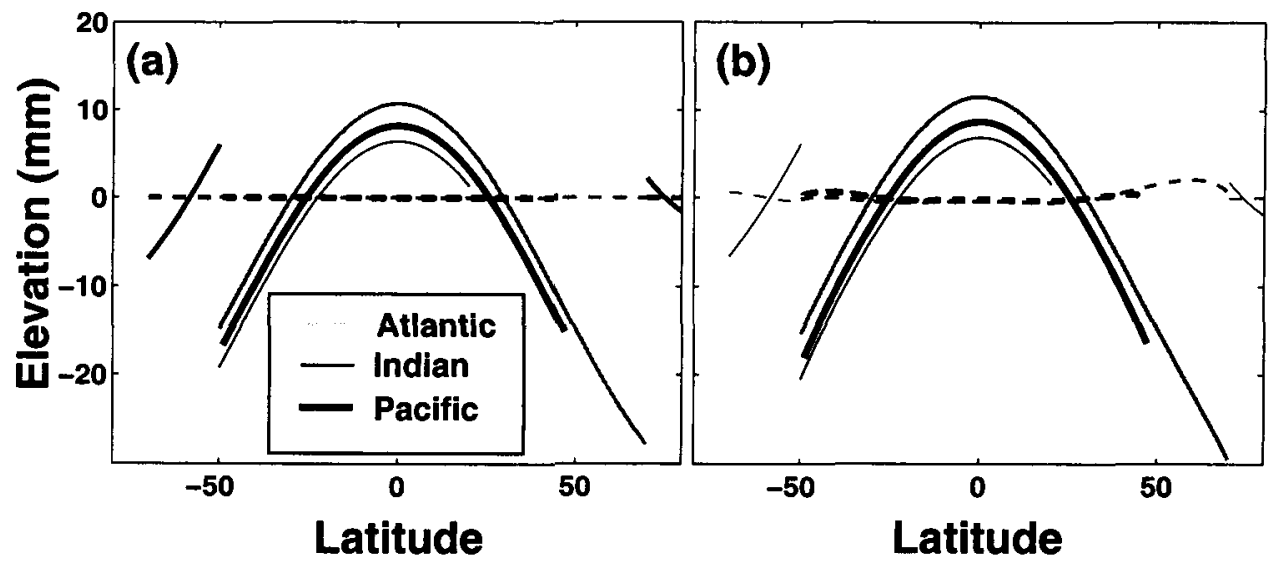

Fig. 4. (a) Self-consistent equilibrium solutions $\hat{\zeta}^{\circ}(\theta)$ for each of the rectangular basins in the schematic model, computed from (4). (b) Zonally averaged numerical solutions $\bar{\zeta}^{\prime \prime}(\theta)$, computed for each of the closed basins forced by the LPT gravitational potential, demonstrating consistency with the self-consistent equilibrium solution of (a). Plotting conventions are as in Fig. 2.

or by flow through the basin mouth, and we then show how solutions for these two special cases can be combined to synthesize the global response.

\section{a. Self-consistent equilibrium solutions in a closed basin}

Because we are interested in the mean basin-scale tidal responses, we begin by considering a closed basin having the simplest possible response: a "self-consistent" equilibrium tide [our use of this term here does not agree exactly with Agnew and Farrell (1978), because we require only that mass be conserved, and do not allow for loading and self-attraction]. All of the schematic basins of Fig. 3 are spherical rectangles bounded by meridians $\phi_{1}, \phi_{2}$, and latitudes $\theta_{1}, \theta_{2}$. With $a$ the radius of the earth, $L=a\left(\theta_{2}-\theta_{1}\right)$ is the northsouth dimension of the basin, and $A=a^{2}\left(\phi_{2}-\phi_{1}\right)$ $\left(\sin \theta_{2}-\sin \theta_{1}\right)$ is the basin surface area. For this geometry the self-consistent equilibrium approximation to the closed basin solution can be given explicitly as

$$
\hat{\zeta}^{(1}(\theta)=\zeta_{\mathrm{EQ}}(\theta)-\frac{\alpha\left[1-\left(\sin ^{3} \theta_{2}-\sin ^{3} \theta_{1}\right)\right]}{2\left(\sin \theta_{2}-\sin \theta_{1}\right)},
$$

where $\zeta_{\mathrm{EQ}}$ is given in $(1)$. The $\hat{\zeta}^{0}(\theta)$ is plotted in Fig. $4 \mathrm{a}$ for each of the five basins in the schematic model. Agreement with zonal averages $\bar{\zeta}(\theta)$ of numerical solutions to (2)-(3) for the five closed basins is excellent (Fig. 4b). Moreover, these numerical results are relatively insensitive to adopted friction (assuming friction is sufficient for numerical convergence). Numerical experiments show that, while resonant Rossby waves can be generated as friction is reduced, the waves have little effect on the zonally averaged elevation. The simple expression (4) thus provides an adequate first approximation to the forced closed-basin response for our schematic ocean.

\section{b. Response of a closed basin to uniform inflow}

At the boundaries with the high-latitude Southern and Arctic Oceans there are significant offsets in the self-consistent equilibrium elevations (see Fig. 4), and these will drive mass flux between basins. The adjustment of elevations across these boundaries will depend on the elevation response to forcing by mass flux through the basin mouth. Consistent with our focus on the large-scale zonally averaged elevations, we consider the response of a rectangular basin to spatially uniform low-frequency flow through a zonal boundary of length $l=a\left(\phi_{2}-\phi_{1}\right) \cos \theta_{1}$ at the southern (or northern) edge of the domain, with no forcing by the LPT potential. A superscript $V$ is used to denote solutions for these forcing and boundary conditions.

As an example, a numerical solution to (2)-(3) for the schematic Pacific basin, $\left(\theta_{1}=-50^{\circ}, \theta_{2}=48^{\circ}\right.$, $\phi_{1}=147^{\circ}, \phi_{2}=272^{\circ}$ ) with $\zeta_{\mathrm{EQ}} \equiv 0$, and unit magnitude periodic normal flow through the south boundary $\left(V \equiv V_{B}=1 \mathrm{~m}^{2} \mathrm{~s}^{-1}\right)$ is shown in Fig. 5. The inphase part of the elevation $\zeta^{v}$ is nearly zero except in boundary layers on the southern and western edges of the basin, where sea levels reach 10-15 mm. The quadrature component of $\zeta^{v}$ is generally larger and nearly uniform $(\approx-12 \mathrm{~mm})$ over the basin. The velocity components are dominantly in-phase with the forcing, and are largest in the boundary layers on the southern and western edges. More detailed examination of numerical solutions at the Mf frequency for this simple basin geometry reveal a number of finerscale features, particularly if the linear friction coefficient is reduced. For example, Rossby waves become prominent with reduced friction. However, as we shall now show, the essential features of the zonally averaged elevation response can be understood in terms of kinematics and geostrophy. More complex 

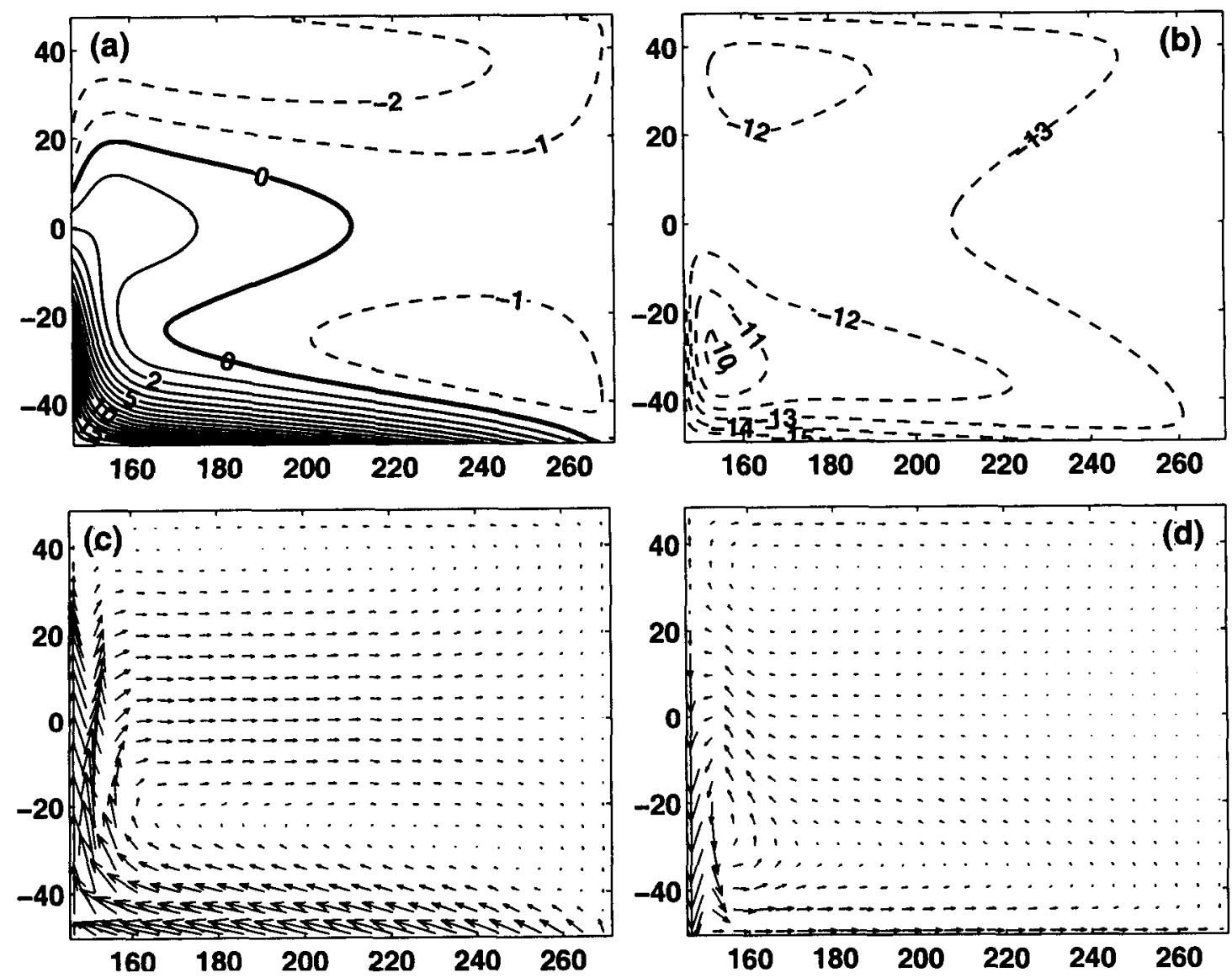

FIG. 5. Solution of (2)-(3) in an idealized, constant-depth (4000 m) Pacific basin, forced by uniform flow through the south boundary. Real and imaginary parts of elevations are given in (a) and (b). Contour interval is $1 \mathrm{~mm}$. Volume transport vectors are plotted in (c) and (d), again as real and imaginary parts. Phase convention is such that (b), (d) occur a quarter period before (a), (c).

dynamics, and in particular wave propagation, are not important to the large-scale elevation response within the basin.

This is most clearly demonstrated by considering the low frequency limit of the basin response. In the appendix we show that to first order in $\omega$, the elevation resulting from forcing by volume flux of constant magnitude $V_{B}$ through a boundary segment of length $l$ takes the form

$$
\zeta^{v}(\theta, \phi ; \omega) \approx \eta_{1}(\theta, \phi)-i l V_{B} /(\omega A)
$$

where $\eta_{1}(\theta, \phi)$ is real and independent of $\omega$, while the imaginary part of $\zeta^{v}$ is a constant inversely proportional to frequency. Equation (5) is readily understood. The time rate of change of the basin average elevation $i \omega\left\langle\zeta^{v}\right\rangle$ must equal the total volume flux $l V_{B}$ across the boundary, divided by the surface area $A$ of the basin. The average elevation thus must be in quadrature to the net inflow, with an amplitude inversely proportional to frequency. At low frequencies volume transports settle down to a frequency-independent form required to distribute the quasi-steady inflow uniformly throughout the basin, while maintaining approximate geostrophic bal- ance with spatial variations in $\zeta^{\nu}$. As frequency is decreased these currents must become increasingly inphase with the forcing at the boundary. Thus, deviations of elevations from the constant $\left\langle\zeta^{v}\right\rangle$ must become real (i.e., in-phase with the normal flow BC) and independent of frequency as $\omega \rightarrow 0$.

Equation (5) was verified further by solving (2)-(3) in the schematic Pacific basin for a series of decreasing frequencies. For $\omega=10^{-7} \mathrm{rad} \mathrm{s}^{-1}$ (corresponding to a period of approximately $2 \mathrm{yr}$ ) the imaginary part of $\zeta^{v}$ is indeed almost constant, with the maximum deviation over the domain from the predicted average about one part in $10^{4}$, and currents are almost exactly in phase. The real part of $\zeta^{v}$ for $\omega=10^{-7} \mathrm{rad} \mathrm{s}^{-1}$ is very close to the results of Fig. 5a, computed at the Mf frequency. Since the imaginary part of $\zeta^{v}$ at the Mf frequency is already nearly constant [with an average that must equal $l V_{B} /(\omega A)$ to satisfy mass conservation], we conclude that the low-frequency approximation (5) is quite adequate to account for the elevation response at the Mf frequency. Note that this conclusion holds only if the friction timescale $\kappa^{-1}$ is short compared to $\omega^{-1}$. If this condition does not hold, resonant Rossby waves are 


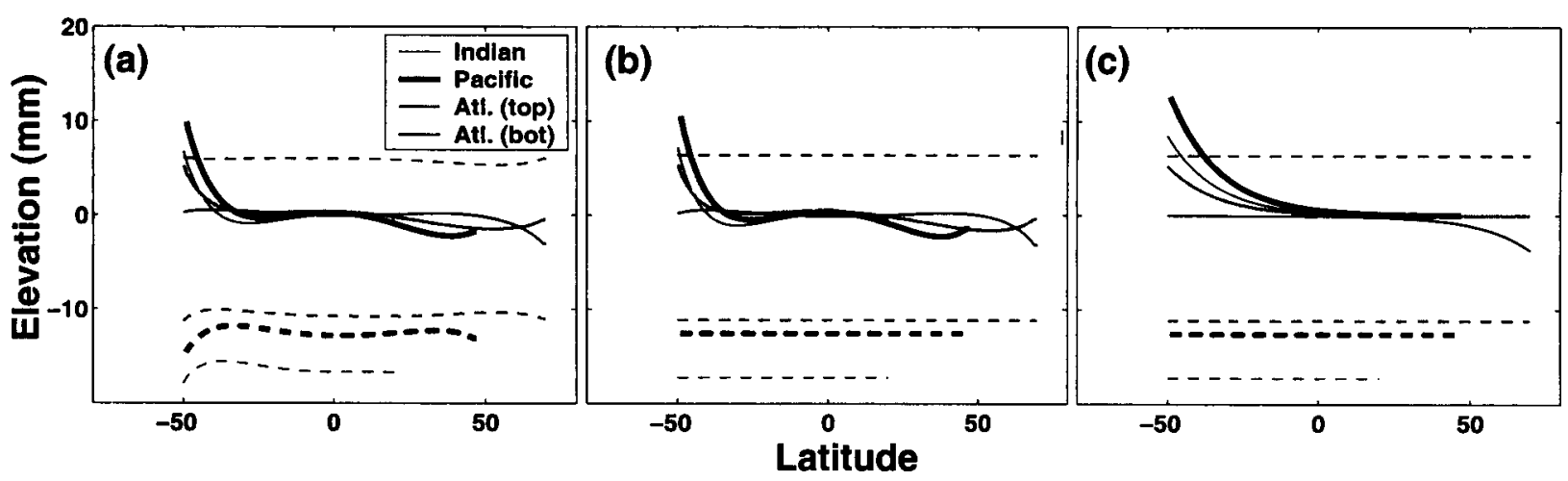

FIG. 6. Zonally averaged elevation response of midlatitude basins to forcing by uniform flow through the mouth: (a) $\bar{\zeta}^{v}(\theta)$ computed by averaging of numerical solutions to the linear shallow-water equations at the Mf frequency; (b) low-frequency approximation computed by zonally averaging (5), with $\eta_{1}$ obtained from a numerical solution to (2)-(3) with $\omega=10^{-7} \mathrm{~s}^{-1}$ and $\zeta_{\mathrm{EQ}}=0$; and (c) analytical approximation $\hat{\zeta}^{v}(\theta)$ computed using $(9)$.

excited, and these will depend on frequency. The spatial dependence of $\eta_{1}$ can be obtained from calculations at any sufficiently low frequency, or by solving a slightly modified steady-state problem (see the appendix).

Zonally averaged elevations $\bar{\zeta}^{v}(\theta)$ from numerical solutions at the Mf frequency in the Pacific, Indian, and Atlantic basins are shown in Fig. 6a, with separate curves corresponding to forcing by flow through the southern and northern boundaries of the Atlantic. Lowfrequency approximations to these elevation profiles $\left[\bar{\eta}_{1}(\theta)-i l /(\omega A)\right]$, are shown in Fig. 6b. Agreement with the actual Mf numerical solutions is very good, although there are some small differences, most noticeably in the imaginary part of the elevations near the inflow boundaries. The approximation (5) involves dynamics only weakly, and in an essentially degenerate sense. Frequency appears only in the imaginary part, which can be derived purely from consideration of mass conservation. The real part $\eta_{1}(\theta, \phi)$ gives the frequency-independent deformation of the free surface required to geostrophically balance the quasi-steady currents, which match the inflow boundary conditions and distribute fluid uniformly throughout the basin.

In the appendix we develop an approximate theory for the low-frequency basin response, valid in the lowfriction limit $\omega \ll \kappa \ll f_{0}$, where $f_{0}$ is the Coriolis parameter at the basin mouth. This analysis shows that there will be boundary layers along the southern, western, and northern edges of the domain. In the boundary layer along the southern edge (the only one that is directly relevant here) $\eta_{1}$ satisfies a one-dimensional diffusion equation, with $-y$ (distance west of the basin edge) playing the role of time. The dynamics are identical to those of the arrested topographic wave discussed by Csanady (1978; see also Winant 1979) in a coastal context. In our much larger-scale problem meridional variations of $f$, rather than cross-shore depth variations, dominate in the conservation of potential vorticity. By analogy with Csanady's terminology, the solution in the boundary layer along the southern edge in Fig. 5 might thus be called an "arrested Rossby wave." An appropriate meridional scale for the boundary layer is

$$
\delta=(L \kappa / \beta)^{1 / 2}
$$

where $\beta=a^{-1} f_{\theta}$ is the usual meridional derivative of the Coriolis parameter (see the appendix).

Following Csanady (1978) and Winant (1979) approximate analytical expressions for $\bar{\eta}_{1}(\theta)$ could be derived, but the calculation is rather messy and the details of the meridional profile across the the boundary layer are not essential for our purposes. Instead we use the very rough approximate form

$$
\bar{\eta}_{1}(\theta) \approx z_{1} \exp \left[-a\left(\theta-\theta_{1}\right) / \delta\right] .
$$

We can directly estimate $z_{1}$, the zonally averaged elevation change across the inflow boundary, by assuming that the zonal volume flux through this boundary layer is equal to the total volume flux into the basin-that is, that the total water mass required to raise and lower the interior of the basin flows zonally through the boundary layer and then out the west end (for the geometry of the Pacific basin of Fig. 5). We show in the appendix that currents in the boundary layer are in geostrophic balance, so the change in elevation across the layer at a point $\phi$ along the lower edge, $\Delta \zeta(\phi)$, is related to the total transport in the layer past this point as $U_{T}(\phi)=$ $-g H \Delta \zeta(\phi) / f$. For uniform inflow $\Delta \zeta(\phi)$ and $U_{T}(\phi)$ will vary linearly for $\phi_{1}<\phi<\phi_{2}$ (see the appendix), so that the average offset in elevation is $z_{1} \approx \Delta \zeta\left[\left(\phi_{2}+\right.\right.$ $\left.\left.\phi_{1}\right) / 2\right]$, and the total volume flux through the west end of the boundary layer is thus $U_{T}\left(\phi_{2}\right) \approx-2 g H z_{1} / f$. This must provide the mass for the rise and fall of the basin. We thus have (for $V_{B}=1 \mathrm{~m}^{2} \mathrm{~s}^{-1}$ )

$$
\frac{-2 g H}{f} z_{1} \approx i \omega A \bar{z}=l V_{B}=l .
$$

Solving for $z_{1}$ and using (5) we obtain a simple approximate analytic expression for $\bar{\zeta}^{v}(\theta)$ : 
$\hat{\zeta}^{v}(\theta)=\frac{f l}{2 g H} \exp \left[-\frac{a\left(\theta-\theta_{1}\right)}{\delta}\right]-i \frac{l}{\omega A} \approx \bar{\zeta}^{v}(\theta)$.

Equation (9) depends essentially on only the gross geometry of the basin and the LPT frequency. The assumed friction coefficient apparently enters as well in defining the boundary layer thickness. However, this aspect of the basin response is of secondary importance, since the large scale variations of elevation between basins will depend only on the elevation offset $z_{1}$.

Applying (9) to the main basins of our schematic ocean model yields the results shown in Fig. 6c. Although there are some consistent differences from the curves computed by averaging numerical solutionsfor example, the actual boundary layers for the numerical solutions (Figs. 6a,b) are all somewhat narrower, and the true $\bar{\eta}_{1}$ always has a small peak at the equatorthe simple analytical expression (9) reproduces the essential features of the zonally averaged basin responses quite well.

\section{c. Interconnected basins}

The zonally averaged elevation profiles of Fig. $6 \mathrm{im}$ ply that interbasin mass flux driven by the LPT potential will only partially equalize sea levels. If there is flow into a basin, geostrophically balanced currents in a zonal boundary layer at the mouth will allow at least some offset to persist. In the global ocean the amplitude and phase of these offsets will be determined by the mutual interaction among all of the interconnected basins. To model this we adapt the theory of Garrett (1975) for tides in a gulf connected to the open ocean to treat the case of multiple interconnected basins of similar size. We only sketch this theory here.

Let $G_{i j}\left(\theta, \phi ; \phi^{\prime}\right)$ be the elevation component of the tidal solution in basin $i$ forced by a delta function in the normal flow at point $\phi^{\prime}$ along the boundary $\mathcal{B}_{i j}$ between basins $i$ and $j$ [i.e., $G_{i j}$ is a Green's function for (2)-(3)]. Let $V_{i j}(\phi), \phi \in \mathcal{B}_{i j}$ be the volume flux on this boundary segment, and let $J_{i}$ be the set of all indices $j$ for which basin $i$ shares a boundary with basin $j$. Then, following Garrett (1975) we readily derive conditions for flow on the basin boundaries, which determine the global solution

$$
\begin{aligned}
& \sum_{k \in J_{i}} \int_{\mathcal{B}_{i k}} G_{i k}\left(\theta_{i j}, \phi ; \phi^{\prime}\right) V_{i k}\left(\phi^{\prime}\right) d \phi^{\prime} \\
& \quad-\sum_{k \in J_{i}} \int_{\mathcal{B}_{j k}} G_{j k}\left(\theta_{i j}, \phi ; \phi^{\prime}\right) V_{j k}\left(\phi^{\prime}\right) d \phi^{\prime} \\
& \quad=\zeta_{j}^{0}\left(\theta_{i j}, \phi\right)-\zeta_{i}^{0}\left(\theta_{i j}, \phi\right) .
\end{aligned}
$$

Here the right-hand side is the offset in tidal elevation on $\mathcal{B}_{i j}$ when a wall exists along this boundary and the tides in each basin are forced by the LPT gravitational potential. The left-hand side is the difference in ele- vation produced on the same boundary by the normal flux through all open boundaries of basins $i$ and $j$. For example, with basins as numbered in Fig. 3, for the Indian Ocean $(i=1)$ elevation at the boundary with the Southern Ocean $(j=4$, so the boundary segment is denoted $\left.\mathcal{B}_{14}\right)$ is determined by flow $V_{14}(\phi)$ at this boundary alone. However the elevation on the south side of $\mathcal{B}_{14}$ is determined by flow on all boundaries of basin $4\left(\mathcal{B}_{41}, \mathcal{B}_{42}, \mathcal{B}_{43}\right)$, so there are three terms in the second summation of (10) for this boundary. In general, taking all of the $N_{b}$ distinct boundary segments $\mathcal{B}_{i j}$ we obtain a set of $N_{b}$ coupled (one dimensional) integral equations which can be solved jointly for the transports $V_{i j}(\phi)$ on all boundaries.

Equation (10) involves no approximations but is too complicated for our purposes. Consistent with our focus on basinwide scales, and with the analysis of individual basins discussed above, we make two approximations. First, we zonally average elevations and transports in each basin, reducing (10) to

$$
\begin{aligned}
& \sum_{k \in J_{i}} \int_{\mathcal{B}_{i k}} \bar{G}_{i k}\left(\theta_{i j}, \phi^{\prime}\right) V_{i k}\left(\phi^{\prime}\right) d \phi^{\prime} \\
& -\sum_{k \in J_{f}} \int_{\mathcal{B}_{j k}} \bar{G}_{j k}\left(\theta_{i j}, \phi^{\prime}\right) V_{j k}\left(\phi^{\prime}\right) d \phi^{\prime} \\
& \quad=\bar{\zeta}_{j}^{0}\left(\theta_{i j}\right)-\bar{\zeta}_{i}^{0}\left(\theta_{i j}\right) .
\end{aligned}
$$

Second, we assume that the LPT fields (at least in the schematic constant depth model) are of sufficiently large scale that we can approximate the integral over boundary segment $\mathcal{B}_{i j}$ as

$$
\begin{aligned}
\int_{\mathcal{B}_{i j}} \bar{G}_{i j}\left(\theta ; \phi^{\prime}\right) V_{i j}\left(\phi^{\prime}\right) d \phi^{\prime} & \approx \bar{V}_{i j} \int_{\mathcal{B}_{i j}} \bar{G}_{i j}\left(\theta ; \phi^{\prime}\right) d \phi^{\prime} \\
& =\bar{V}_{i j} \bar{\zeta}_{i j}^{V}(\theta) .
\end{aligned}
$$

Here, $\bar{\zeta}_{i j}^{V}$ is just the zonal average of the unforced solution to (2)-(3) with unit magnitude normal flow boundary condition on $\mathcal{B}_{i j}$. The approximation (12) can be justified a priori by our analysis of the closed basin solution, which showed that the zonally averaged offset at the basin mouth is determined kinematically, by the total flow into the basin. The approximation is justified further below, where we show that solutions derived under this approximation are in very good agreement with a full numerical solution.

Using (12) and defining

$$
Z_{i j}^{i k}=\frac{\int_{\mathcal{B}_{i j}} \int_{\mathcal{B}_{i k}} G_{i j}\left(\theta_{i k}, \phi ; \theta_{i j}, \phi^{\prime}\right) d \phi d \phi^{\prime}}{\int_{\mathcal{B}_{i k}} d \phi},
$$

we can approximate the coupled system of integral equations (10) by a system of $N_{b}$ ordinary linear equations 
in $N_{b}$ unknowns $\bar{V}_{i k}$, representing the zonally averaged volume fluxes across each basin boundary:

$$
\sum_{k \in I_{i}} Z_{i k}^{i j} \bar{V}_{i k}-\sum_{k \in J_{j}} Z_{j k}^{j i} \bar{V}_{j k}=\bar{\zeta}_{j}^{0}\left(\theta_{i j}\right)-\bar{\zeta}_{i}^{0}\left(\theta_{i j}\right)
$$

With these simplifications, the zonally averaged solution in basin $i$ is approximated as

$$
\bar{\zeta}_{i}(\theta)=\bar{\zeta}_{i}^{0}(\theta)+\sum_{j \in J_{i}} \bar{V}_{i j} \bar{\zeta}_{i j}^{v}(\theta)
$$

Here $Z_{i j}^{i k}$ is the average $\zeta^{v}$ in basin $i$ on boundary segment $i k$ due to unit normal flow forcing everywhere on boundary segment $i j$. In the simplest case with only a single connecting boundary (e.g., the Indian or Pacific basins in our schematic model) only $Z_{i j}^{i j}$ is defined. This single complex number can be interpreted as an impedance, which gives the amplitude and phase of the elevation at the basin mouth (comparable to voltage) resulting from a unit magnitude oscillating current input (Garrett 1975). Impedances for the Pacific, Indian, and Atlantic can be read off Fig. 6; that is, they are the zonally averaged complex elevations evaluated at the inflow boundaries. Note that for the Atlantic there are two inflow boundaries, and there are a total of four impedance elements, corresponding to elevations at the north and south boundaries due to flow through each. For the Southern Ocean the situation is even more complicated, as one must account for the average elevation at each basin mouth from constant forcing at each of the three boundaries (i.e., in this case there are nine impedance elements).

For the schematic model with basins numbered as in Fig. 3, there are $N_{b}=4$ basin boundaries, and the linear system (14) is given explicitly by

$$
\left[\begin{array}{ccc}
Z_{14}^{14}-Z_{41}^{41} & -Z_{42}^{41} & -Z_{43}^{41} \\
-Z_{41}^{42} & Z_{24}^{24}-Z_{42}^{41} & -Z_{43}^{42} \\
-Z_{41}^{43} & -Z_{42}^{43} & Z_{34}^{34}-Z_{43}^{42} \\
0 & 0 & Z_{34}^{35}
\end{array}\right.
$$

To apply (14)-(15) we need the zonally averaged responses to LPT forcing $\left(\bar{\zeta}_{i}^{0}\right)$ and to normal flow at all basin boundaries $\left[\bar{\zeta}_{i j}^{V}(\theta)\right]$, and the impedance elements $Z_{i j}^{k}$. All of these can be computed directly by numerically solving the shallow-water equations for each basin with appropriate forcing and boundary conditions, and then zonally averaging. Results from this numerical approach have already been given in Fig. $4 \mathrm{~b}\left(\bar{\zeta}_{i}^{0}\right)$, and Fig. 6a $\left[\bar{\zeta}_{i j}^{V}(\theta)\right]$. As noted above, the complex impedances $Z_{i j}^{i j}, i$ $=1,2,3 ; j=4,5$; and $Z_{35}^{34}$ and $Z_{34}^{35}$ (i.e., for the Indian, Pacific, and Atlantic at boundaries with the Southern and Arctic Oceans) can be read off of Fig. 6a. The remaining impedances $Z_{i j}^{i k}$ required for (16) can be obtained by averaging the two-dimensional solutions for the Arctic and Southern Oceans over each of the appropriate basin boundaries as in (13). Note that for the Southern Ocean separate solutions must be computed for forcing by flow at each of the three boundary segments.

Using these impedances in (14) and solving for the average interbasin fluxes $\bar{V}_{i j}$, we use (15) to obtain the zonally averaged elevations $\bar{\zeta}_{i}(\theta)$ shown in Fig. $7 \mathrm{a}$. The results are nearly identical to the actual zonal averages computed from the global two-dimensional numerical model (cf. to Fig. 2c), verifying a posteriori that zonal averaging of forcings and responses to reduce the system of integral equations of (10) to the small system of discrete linear equations (14) is valid.

Except for the Southern and Arctic Oceans, (9) pro-

$$
\left.\begin{array}{c}
0 \\
Z_{35}^{34} \\
Z_{35}^{35}-Z_{53}^{53}
\end{array}\right]\left[\begin{array}{l}
\bar{V}_{14} \\
\bar{V}_{24} \\
\bar{V}_{34} \\
\bar{V}_{35}
\end{array}\right]=\left[\begin{array}{l}
\bar{\zeta}_{4}^{0}\left(\theta_{14}\right)-\bar{\zeta}_{1}^{0}\left(\theta_{14}\right) \\
\bar{\zeta}_{4}^{0}\left(\theta_{24}\right)-\bar{\zeta}_{2}^{0}\left(\theta_{24}\right) \\
\bar{\zeta}_{4}^{0}\left(\theta_{34}\right)-\bar{\zeta}_{3}^{0}\left(\theta_{34}\right) \\
\bar{\zeta}_{5}^{0}\left(\theta_{35}\right)-\bar{\zeta}_{3}^{0}\left(\theta_{35}\right)
\end{array}\right] .
$$

vides a simple analytical approximation for $\bar{\zeta}_{i j}^{v}(\theta)$ and for most of the impedances $Z_{i j}^{i k}$. To estimate the remaining impedances used in (16), we need expressions for the responses of the zonally periodic Southern and Arctic Ocean basins to forcing by flow through only a portion of the zonal boundary. For these we assume that the elevation in the vicinity of the inflow is also of the form (9), but with $l$ now giving the length of this boundary segment. Elsewhere in the basin (in particular on boundary segments where "off-diagonal" impedances must be evaluated) we assume that the elevation is adequately approximated by the spatially constant imaginary part of (9). The same approximation is used to estimate $\bar{\zeta}_{i j}^{v}(\theta)$ for these high-latitude basins. In fact, two-dimensional numerical calculations show that the low-frequency geostrophically balanced elevation perturbation $\eta_{1}(\theta, \phi)$ resulting from flow through a portion of the zonal boundary extends well beyond the open segment. This last approximation is thus probably the biggest source of error in our purely analytic approximate model.

Using the approximate impedances to construct the coefficient matrix $\mathbf{Z}$ and the self-consistent equilibrium approximations of (4) to form the right-hand side of (16), we can solve for transports across each basin boundary $\bar{V}_{i j}$. These can then be used in (15), with the analytical approximations $\hat{\zeta}_{i j}^{v}(\theta)$ and $\hat{\zeta}_{i}^{0}(\theta)$ of (9) and (4), to estimate the zonally averaged elevations in each basin. The result, given in Fig. $7 b$, is close to the results 
obtained from the full two-dimensional schematic model (Fig. 2c; see also Fig. 7a), and is a reasonable approximation to the zonally averaged empirical T/P Mf elevations (Fig. 2a).

\section{Discussion}

We have shown that the large-scale nonequilibrium part of Mf can be reproduced by a very simple, almost kinematic model. Individual closed basins would by themselves have mean elevations that differ from static equilibrium only by a constant, determined by the condition that mass be conserved. The resulting offsets between connected basins force interbasin flows, which can be computed by requiring elevations to match across boundaries. Interbasin flows can thus be computed once individual basin "impedances" (the ratio of elevation at the basin mouth to volume flux) are known. These fluxes, together with the associated meridional profile of elevations, allow computation of interbasin differences in LPT elevations. Drawing on two-dimensional numerical modeling results and a scaling analysis of the shallow-water equations, we have developed a simple approximate model for the low-frequency response of a basin to flow across the north or south boundary. In the low-frequency limit elevations are nearly equal to a purely imaginary constant $\bar{\zeta}^{v}(=-i l / \omega A)$, describing a uniform basinwide rise and fall of sea level in quadrature to the inflow. As $\omega \rightarrow 0$, flow in the basin approaches a fixed limit, in phase with the flow across the boundary and concentrated in a narrow zone of geostrophically balanced currents along the mouth and western edge. The large-scale global equilibrium response depends only on the basinwide average elevation $\bar{z}$ and the average vertical offset across the boundary layer $z_{1}$, and both of these constants can be estimated from simple considerations of mass conservation (and geostrophy).

If the ratio

$$
z_{1} / \bar{z}=-i \omega f A l / 2 g H
$$

is significant, offsets (over a layer of width $\delta$ ) between the basin interiors are allowed, and the tide will not generally be in global equilibrium. As this ratio goes to zero (e.g., as $\omega \rightarrow 0$ ), the global self-consistent equilibrium form will be approached.

The linear dependence of $z_{1} / \bar{z}$ on frequency is a simple, but powerful, description of the large-scale response of the ocean and merits confirmation (cf. Proudman 1960). An obvious way to proceed is to examine explicitly the ocean's response at other frequencies, and an obvious frequency to consider is that of the lunar monthly tide $\mathrm{Mm}$. The zonally averaged ocean response at the $\mathrm{Mm}$ frequency (and for the Mm amplitude) computed with the simple analytical model is shown in Fig. $8 \mathrm{a}$. It is indeed noticeably closer to equilibrium than the comparable Mf results (Fig. 7b). Numerical solution of the shallow water equations for the schematic flat-bottom ocean model yields very similar results (Fig. 8b).
Basinwide zonal averages of $\mathrm{Mm}$ estimated from $\mathrm{T} / \mathrm{P}$ altimeter data (Fig. 8c) are also closer to equilibrium than the empirical Mf estimates of Fig. 2a. While the empirical estimates for both $\mathrm{Mf}$ and $\mathrm{Mm}$ do deviate significantly more from equilibrium than the corresponding results for the schematic ocean basins, the main basin-scale pattern, and its dependence on frequency, is captured well by even our simplest model.

To explore further the frequency dependence of the nonequilibrium response we solved the linear shallowwater equations for the schematic flat-bottom ocean of Figs. le-1f for a range of nontidal frequencies, in all cases forced with the Mf gravitational potential. Solutions were obtained with two linear friction coefficients: the high friction case we have considered so far $(r=$ 0.03 ), and a low-friction case with $r=0.001$. To summarize results for all of these calculations we plot the difference between zonal averages at the equator in the Pacific and Atlantic basins in Fig. 9. At the lower frequencies relevant to the LPT, our simplified analytical model reproduces the frequency dependence of both amplitude and phase of the Atlantic-Pacific equatorial difference quite well. Both the analytical and numerical models show that the interbasin elevation differences go to zero at low frequencies, with a reduction in the amplitude of the nonequilibrium response by roughly a factor of 2 for $\mathrm{Mm}$ relative to $\mathrm{Mf}$.

For frequencies below about $0.1 \mathrm{cpd}$ there is essentially no difference in Fig. 9 between the high and low friction cases. This is consistent with our analytical results, which show that friction should enter only in determining the width of the boundary layer over which elevation differences are accommodated. Insensitivity to dissipation is further confirmed in Fig. 10a, which shows zonal averages of a numerical solution for the schematic model, computed with the smaller friction coefficient $(r=0.001)$. The large-scale pattern in midlatitude basin interiors is changed little from the standard high friction case (Fig. 2c). Yet aside from these basin-scale patterns, there are in fact significant differences between the high- and low-friction solutions. In particular, with $r=0.03$ there is no evidence for Rossby waves in the elevations (Figs. 1e-f), nor in the corresponding currents (not shown), while the low-friction solution has large-amplitude Rossby waves in all basins. Thus, at LPT frequencies friction can significantly damp resonant Rossby waves, but it evidently has minimal effect on the large-scale pattern of interbasin elevation variations. These large-scale interbasin LPT elevation variations will thus not be particularly informative about the dissipation of low-frequency barotropic ocean motions.

More significant changes in elevations occur in the Arctic and Southern Oceans when friction is reduced, suggesting that LPT elevations in these basins are likely to be more directly informative about low-frequency barotropic dissipation than are the basin-scale elevation deviations we have focused on. In fact, model results 

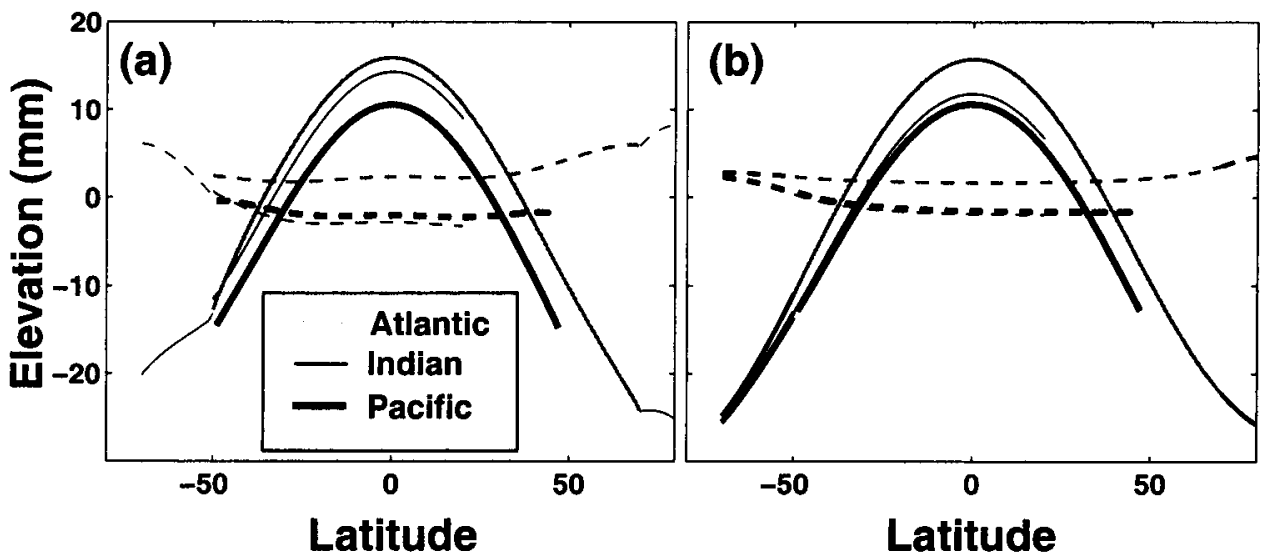

FIG. 7. Estimates of global (zonally averaged) Mf response from (2)-(3) (a) with $\bar{\zeta}_{i}^{0}, \bar{\zeta}_{i j}^{v}$, and the impedances $Z_{i k}^{j i}$ computed by zonally averaging numerical solutions to the shallow-water equations, and (b) with the corresponding analytical approximations $\left(\hat{\zeta}_{i}^{o}, \hat{\zeta}_{i j}^{V}\right.$, and the corresponding impedances).

for the high-friction case (Fig. $2 \mathrm{c}$ ) more closely match the empirical T/P estimates (Fig. 2a), suggesting a relatively high value for effective dissipation, but this issue needs further study.

For higher frequencies $(>0.1 \mathrm{cpd})$ friction does have a significant effect on the zonally averaged elevations, as the rapid variation with frequency of equatorial elevation differences in Fig. 9 shows. These rapid variations result from resonant normal modes, a topic which has been raised in a number of previous discussions of the LPT (Wunsch 1967; Luther 1980; Miller et al. 1993; Wunsch et al. 1997). Over the full frequency band of Fig. 9 there surely exist many oceanic normal modes. For a water covered earth with constant $(\approx 4000 \mathrm{~m}$ ) depth, gravitational and vorticity modes are divided into two cleanly separated branches, with an accumulation point at zero frequency for vorticity modes and a gravest gravitational mode with a period of around 1 day (e.g., Longuet-Higgins 1968; Marchuk and Kagan 1989). For realistic ocean basin geometries, Platzman et al.
(1981) found no modes of gravitational character with periods of more than about $40 \mathrm{~h}$, and Gotlib et al. (1987; see also Marchuk and Kagan 1989) report that the gravest gravitational mode has a period of about $100 \mathrm{~h}(0.25 \mathrm{cpd})$. Below $0.1 \mathrm{cpd}$ modes are almost certainly dominated by vorticity, have relatively short spatial length scales, and as a consequence probably have little effect on large-scale interbasin elevation variations.

Whether individual modes are evident in the ocean depends in part on whether they are sufficiently sharp (sufficiently high $Q$ ) in the vicinity of forcing frequencies, which depends, of course, on dissipation. For a 4000-m ocean the two friction coefficients used in Fig. 9 correspond to timescales of $T_{f}=50$ days $(r=0.001)$ and $T_{f}=1.5$ days $(r=0.03)$, implying a resolution in frequency of about $0.003 \mathrm{cpd}$ and $0.1 \mathrm{cpd}$ respectively. In the low-friction case there is clear evidence for normal modes in the frequency band $0.1-0.3 \mathrm{cpd}$. These modes are revealed by the significant variations of interbasin equatorial elevation difference for frequencies
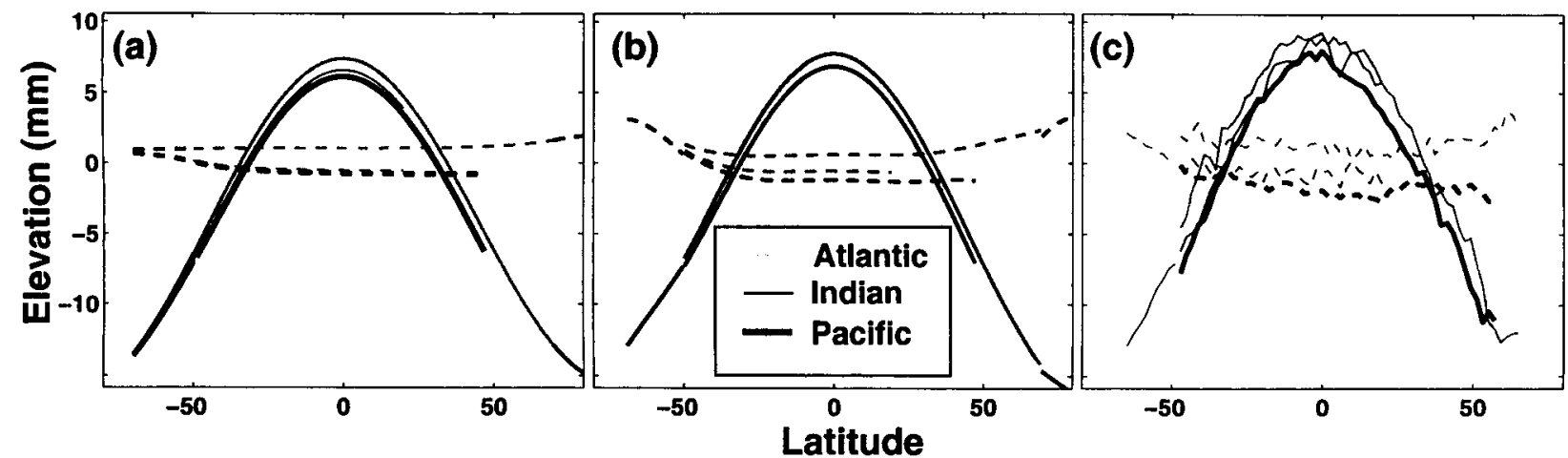

FIG. 8. Zonally averaged Mm solutions, computed from (a) the simple analytical model, (b) numerical solution of the shallow-water equations in a schematic ocean, and (c) averaging the T/P empirical estimates. 

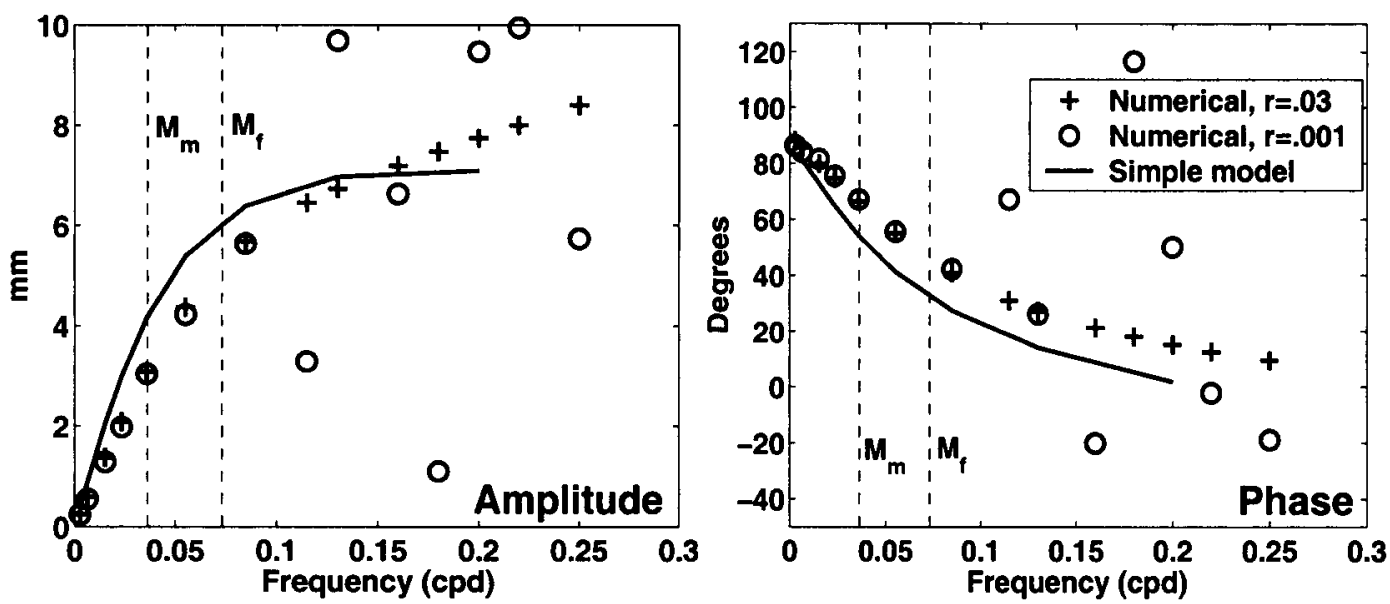

FIG. 9. Amplitude and phase of the (vector) difference between zonally averaged complex elevations at the equator for the Atlantic and Pacific basins, as a function of frequency. Symbols give results from solution of the 2D shallowwater equations for the flat-bottom, rectangular basin geometry of Figs. le-f, using two different values for the linear friction coefficient. Results computed from the simple analytical model are given by the solid line. The same forcing, with amplitude corresponding to the Mf potential, was used in all cases.

separated by more than the inverse dissipation timescale of $0.003 \mathrm{cpd}$. In the dissipative $(r=0.03)$ case (which is perhaps more relevant to the real ocean) the effect of individual resonant modes in this band is completely smoothed out.

In contrast, at frequencies below $0.1 \mathrm{cpd}$ - the regime dominated by vorticity modes-no resonant modes are evident in Fig. 9 for either high or low friction. The elevation response below $0.1 \mathrm{cpd}$ for the low-friction case is smooth over a band that is wide in comparison with the resolution implied by the frictional damping timescale. This implies that resonance of vorticity modes, which must be prevalent within this band, does not contribute significantly to the large-scale elevation response we focus on here. This conclusion is also supported by the weak dependence on friction of the zonal Mf responses obtained from the two-dimensional numerical solutions (Figs. $2 \mathrm{c}$ and $9 \mathrm{a}$ ). Individual vorticity modes near the Mf frequency are probably excited only very weakly in the LPT response because their spatial structure is poorly matched to the curl-free zonal forcing. Any attempt to explain the large-scale nonequilibrium tidal elevation response in terms of nearly resonant Rossby waves, which Wunsch (1967) reasonably proposed for some shorter-scale features in the Pacific Ocean, must clearly be inadequate at these scales. This conclusion is consistent with that of Miller et al. (1993).

Our analysis of the low-frequency response of an individual basin to forcing by flow through a zonal boundary suggests that in the absence of rotation $(f=0)$ the entire basin will rise and fall essentially as one, with no offset at the mouth [see (17)]. Running the twodimensional numerical model with $f=0$ confirms that at the Mf frequency individual basin responses are flat and the global solution is very nearly in equilibrium (Fig. 10b). At somewhat higher frequencies gravita-

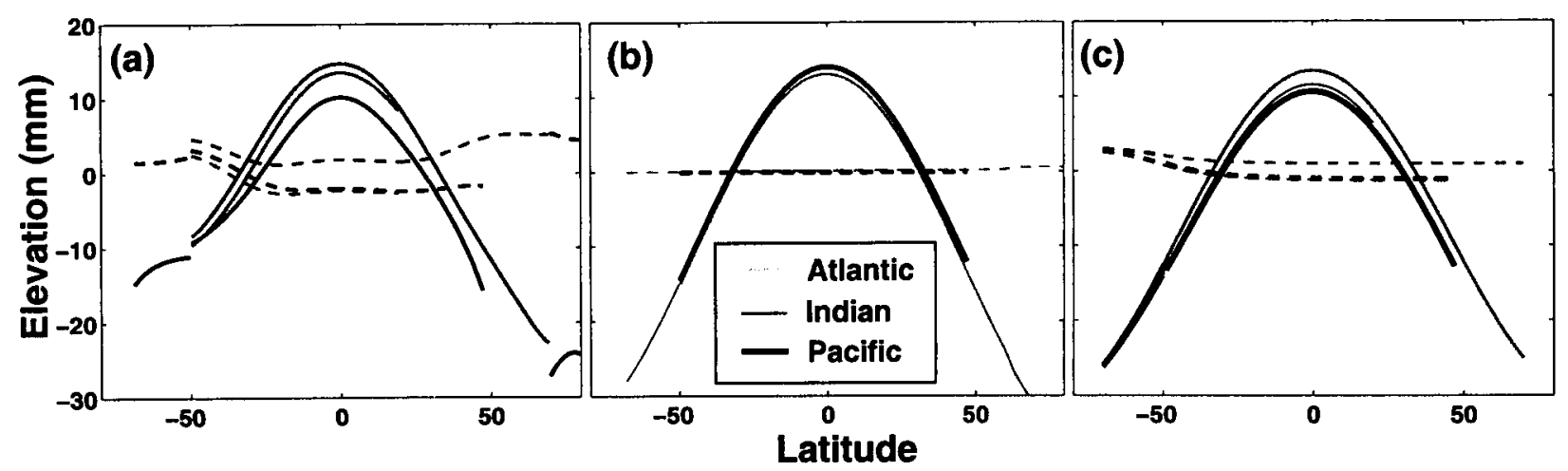

Fig. 10. Zonal averages of Mf elevations for each basin computed from (a) the numerical solution for the schematic ocean, with friction reduced by a factor of 30 ; (b) the numerical solution for the schematic ocean with no rotation; and (c) the simple analytical model, with the Arctic Ocean omitted. 
tional normal modes are more easily excited, and significant deviations from equilibrium can occur; see, for example, Fig. 11 of Ponte (1997), which shows a nonequilibrium global response to large-scale forcing for $f$ $=0$ at periods around 5 days, close to the period of the gravest normal mode found by Gotlib et al. Thus, while low-frequency gravitational modes are almost certainly more compatible with the spatial structure of the LPT forcing, these apparently have resonant frequencies well above that of Mf. We conclude that the LPT response cannot reasonably be explained in terms of resonance of a small number of normal modes, of either gravitational or vorticity character. Thus, as noted by Wunsch et al. (1997), a normal mode framework is not particularly enlightening in this case.

Miller et al. argued that Mf waves generated in the Arctic where the LPT potential is maximum propagate as gravity waves to the Pacific by way of the Atlantic and Indian Oceans. We can easily examine the role of the Arctic with our simplified model. To close the Arctic, we need only eliminate one unknown $\left(\bar{V}_{35}\right)$ and one (the last) equation from (16). The resulting estimate of zonally averaged elevations (Fig. 10c) indeed shows that inter-basin Mf differences are significantly reduced when the Arctic is closed off. Two-dimensional numerical calculations for a schematic global ocean without an Arctic Ocean produce similar results.

Our results confirm the importance of the Arctic, but do not support the conceptual model of Miller et al. The theory for interconnected basins embodied in (16) is essentially just the statement that elevations and mass fluxes must match at basin boundaries. We have further shown that at LPT frequencies a closed basin will be nearly in equilibrium with the gravitational forcing, while the response to forcing by flow through the north or south boundary of the basin is explained well by an essentially kinematic theory, with quasi-static currents in geostrophic balance with deformations of the free surface near the mouth of the basin. No element in this model invokes (or even allows) wave propagation in any manner. Miller et al. (1993) noted that in numerical solutions to the shallow-water equations Mf phase lags in the Pacific increased when $g$ was reduced, but decreased when $f$ was reduced. The first trend is consistent with their interpretation of the LPT response in terms of gravity waves, and the second trend shows that the non-equilibrium response cannot reasonably be explained in terms of propagation of Rossby waves, which would have reduced phase speeds for slower rotation. However, both trends are also predicted by our (17), which is derived from an essentially static kinematic theory. Thus, while the observed progression of Mf phase lags between basins may be suggestive of propagation, it is misleading, in our view, to think of waves being generated in a single basin and then propagating basin to basin around the globe.

We conclude that description of the Mf tide in terms of Rossby or gravity waves does not offer much insight into the cause of the large-scale deviation of elevations from equilibrium. Of course this does not imply that Rossby or gravity waves are inapplicable to the theory of long-period tides. There are many other senses in which the LPT diverge from the simple equilibrium form, and at shorter spatial scales both planetary and topographic Rossby waves are likely to be important. Moreover, LPT currents are of necessity nonequilibrium, and for these quasigeostrophic dynamics and topographic effects must certainly be important.

Our simple model for the nonequilibrium elevation response of the ocean to forcing by the LPT potential may also help to explain some aspects of the oceanic response to atmospheric pressure variations. At periods longer than a few days much of the pressure-driven sea level variability is reasonably isostatic, with the relationship between atmospheric pressure and elevation fairly well approximated by a local inverted barometer response (e.g., Wunsch and Stammer 1997). However, at periods of around 4-6 days deviations from the inverted barometer response are anomalously large (Luther 1982; Woodworth et al. 1995; Ponte 1997; Hirose et al. 2001). Luther (1982) suggested that much of the atmospheric forcing in this band was probably associated with the approximately 5-day Rossby-Haurwitz mode in the atmosphere (Madden and Julian 1972). Luther further suggested that the anomalous deviation from equilibrium in the ocean response might be associated with excitation of one or more barotropic normal modes of the Pacific basin.

Global-scale atmospheric pressure variations, in particular the zonal wavenumber $m=-1$ Rossby-Haurwitz mode, would also be expected to drive flow between basins. The mechanism we propose to explain the nonequilibrium LPT response could thus also help to explain coherent basin-scale deviations from the inverted barometer response for periods around 5 days. We thus suggest that the observed narrow-band anomaly in oceanic response is a reflection of the unusually large spatial scale of the atmospheric forcing near this period, rather than any oceanic resonance. This suggestion is consistent with the conclusions of Ponte (1997) and Hirose et al. (2001), that any oceanic resonance effects must be weak, and that large-scale adjustment of the mass field must play an important role. Using a barotropic ocean model forced by atmospheric pressure variations, Hirose et al. (2001) found a nonequilibrium ocean response that was dominated by step-like offsets between basins, essentially the same pattern we have identified in the nonequilibrium LPT. Because the spatial structure of the Rossby-Haurwitz mode is quite different from the LPT gravitational potential, and because the frequency of this atmospheric forcing is higher where gravitational modes may have a more significant impact on the oceanic response, our simplified analytical model may not be directly applicable in all details. However, it seems clear that the general mechanism developed here will be relevant to any large-scale forcing 
that can drive a significant interbasin mass flux, including in particular the 5-day atmospherically forced response.

We have focused on deviations of LPT elevations from equilibrium at basinwide scales, where kinematics and geostrophy dominate and friction plays a secondary role. At these scales estimates of LPT from altimetry are unlikely to provide useful constraints on the dissipation of low-frequency barotropic ocean motions. However, as altimeter data continue to accumulate, accuracies of empirically estimated LPT elevations will increase, and finer-scale features will be resolved. This will open the possibility for a number of interesting additional studies. Most obviously, what is the nature of the LPT currents? To what extent are these dominated by propagating and trapped Rossby waves? In contrast to the large-scale elevations the amplitudes of these will be sensitive to the effective dissipation, which must depend heavily on stratification and nontidal ocean motions. To the extent that this is true, even barotropic LPT currents may conceivably be quite variable in time. Our analysis here suggests two other possible effects of friction, which might be more readily and directly observable in the altimetry data. First, while dissipation has little effect on low-latitude interbasin elevation differences, it appears to have a more significant effect on deviations of the LPT from equilibrium in the zonally connected Southern Ocean. Second, we have shown that the width of the boundary layers over which basin offsets are accommodated will depend on friction. Careful analysis of the LPT in the Southern Ocean, and of the boundary layer widths at basin offsets, might well provide some constraint on the dissipation of barotropic ocean currents at periods of weeks to months.

Acknowledgments. We thank John Allen for helpful discussions and for encouraging a more careful look at the scaling of boundary layer widths. The paper was significantly improved by reviews from Chris Garrett, who pointed out the connection to arrested topographic waves, and an anonymous referee. This work was partially supported by the National Science Foundation (Grants OCE-9633527 and OCE-9819518 to GDE), and by the National Aeronautics and Space Administration through the TOPEX/Poseidon and Jason-1 projects.

\section{APPENDIX}

\section{Response of a Closed Basin to Flow through a Zonal Boundary}

In this appendix we derive the low-frequency approximation (5) for elevations in a basin forced by flow through the boundary, and we use a scaling analysis of the shallow-water equations to justify more formally the approximate basin response (9).

We consider the linear shallow-water equations (2)(3), initially in a general domain (on the sphere, but our conclusions would hold for the same equations in a planar region, as assumed below). Boundary conditions (BC) are specified normal volume flux on the boundary $\hat{\mathbf{n}} \cdot \mathbf{U}=V_{B}$, where $\hat{\mathbf{n}}$ is the outward-directed normal to the boundary, and $V_{B}$ is a specified real function of position on the boundary $\mathcal{B}$ (i.e., we assume that all flow across the boundary is in phase). The surface area of the basin is denoted by $A$, and the net volume flux is $\mathcal{V}_{T}=\int_{\mathcal{B}} V_{B} d l$.

We first show that as $\omega \rightarrow 0$ the elevation $\zeta$ approaches a limit of the general form

$$
\zeta(\theta, \phi ; \omega)=\frac{i \mathcal{V}_{T}}{\omega A}+\eta_{1}(\theta, \phi),
$$

where $\eta_{1}$ is real and independent of frequency. Averaging $\zeta$ over the model domain, using (3) and Gauss's law we obtain $\langle\zeta\rangle=i \mathcal{V}_{T} / \omega A$. As $\zeta$ becomes unbounded in the low-frequency limit of interest, it is convenient to replace $\zeta$ with $\eta=\omega \zeta$. Then the basinwide average $\langle\eta\rangle=\omega\langle\zeta\rangle=i \mathcal{V}_{T} / A$ is a fixed purely imaginary constant (independent of frequency) and $\mathbf{U}, \boldsymbol{\eta}$ satisfy a slightly modified version of (2)-(3):

$$
\left(\omega \mathbf{F}+i \omega^{2} \mathbf{I}\right) \mathbf{U}=-c^{2} \boldsymbol{\nabla} \quad \boldsymbol{\nabla} \cdot \mathbf{U}=-i \eta,
$$

with the same $\mathrm{BC}$ on $\mathbf{U}$.

Expanding the solution to (A2) at each point in the domain as an asymptotic series in $\omega$

$$
\begin{aligned}
\eta(\theta, \phi ; \omega) & =\eta_{0}(\theta, \phi)+\omega \eta_{1}(\theta, \phi)+\cdots \\
\mathbf{U}(\theta, \phi ; \omega) & =\mathbf{U}_{0}(\theta, \phi)+\omega \mathbf{U}_{1}(\theta, \phi)+\cdots,
\end{aligned}
$$

substituting into (A2), and collecting terms of like powers in $\omega$, we obtain

$$
\begin{aligned}
\boldsymbol{\nabla} \eta_{0} & =0 \\
\boldsymbol{\nabla} \cdot \mathbf{U}_{0} & =-i \eta_{0} \\
\mathbf{F} \mathbf{U}_{0} & =-c^{2} \nabla \eta_{1} .
\end{aligned}
$$

Equation (A5) implies $\eta_{0}$ is constant, so we must have $\eta_{0}(\theta, \phi) \equiv i \mathcal{V}_{T} / A$, and (A6) becomes

$$
\boldsymbol{\nabla} \cdot \mathbf{U}_{0}=\mathcal{V}_{T} / A \text {. }
$$

Equation (A8), together with (A7) and the BC on normal transports (which do not depend on $\omega$, and hence must apply to the limiting zero order velocity $\mathbf{U}_{0}$ ) determine the pair $\mathbf{U}_{0}, \eta_{1}$. Because (A7)-(A8) and the BC are all real, the solution $\eta_{1}$ must also be real. Thus to first order in $\omega$

$$
\begin{aligned}
\zeta(\theta, \phi ; \omega) & =\omega^{-1}\left[\eta_{0}+\omega \eta_{1}(\theta, \phi)\right] \\
& =i \nu_{T} / A \omega+\eta_{1}(\theta, \phi),
\end{aligned}
$$

proving (A I). Note that (A7)-(A8) define a steady-state problem (independent of $\omega$ ), with the spatially uniform source term for the continuity equation compensating for the steady inflow through the southern boundary.

For the rectangular basin with a boundary at the southern edge (as in section $3 \mathrm{~b}$ ), $\mathcal{V}_{T}=-V_{B} l$, establish- 
ing (5). To qualitatively understand the steady-state solution $\eta_{1}(\theta, \phi)$ we consider a scaling analysis of (A7)(A8). To be explicit we assume a rectangular basin in the $x, y$ plane with $y \in[-L, L], x \in[0, L]$, and uniform flow $V_{B}=1$ through the southern edge of the domain (so $A=2 L^{2}$, and $\nu_{T}=L V_{B}$ ). We drop subscripts on $\eta_{1}$ and $\mathbf{U}_{0}$, and to slightly simplify algebra we make a beta-plane approximation $f=\beta y$. A second-order equation for $\eta$ is readily derived by eliminating volume transports. In dimensional form this is

$$
\begin{aligned}
& \frac{\kappa}{\beta}\left(\eta_{x x}+\eta_{y y}\right)+\frac{f^{2}-\kappa^{2}}{f^{2}+\kappa^{2}} \eta_{x}-\frac{2 \kappa f}{\kappa^{2}+f^{2}} \eta_{y} \\
& =\frac{\kappa^{2}+f^{2}}{\beta c^{2}} \frac{V_{B}}{2 L} .
\end{aligned}
$$

To nondimensionalize we take $L$ as the length scale, and the average elevation change across the mouth of the basin (see section 4):

$$
z_{1}=\frac{f_{0} L V_{B}}{2 c^{2}}
$$

where

$$
f_{0}=\beta L,
$$

as a scale for $\eta$. Nondimensionalizing as $(x, y)=L\left(x^{\prime}\right.$, $\left.y^{\prime}\right), \eta=z_{1} \eta^{\prime}$, writing

$$
\boldsymbol{\epsilon}=\kappa / f_{0},
$$

substituting into (A10), simplifying and dropping the primes, we obtain (after noting that in nondimensional units $\left.f / f_{0}=y \in[-1,1]\right)$ :

$$
\begin{aligned}
& \epsilon\left(\eta_{x x}+\eta_{y y}\right)+\frac{y^{2}-\epsilon^{2}}{y^{2}+\epsilon^{2}} \eta_{x}-\frac{2 \epsilon y}{\epsilon^{2}+y^{2}} \eta_{y} \\
& =\epsilon^{2}+y^{2} .
\end{aligned}
$$

In nondimensional form the velocities (scaled by $V_{B}$ ) are determined from

$$
\begin{aligned}
& -2\left(y^{2}+\epsilon^{2}\right) U=\left(\epsilon \eta_{x}+y \eta_{y}\right) \\
& -2\left(y^{2}+\epsilon^{2}\right) V=\left(-y \eta_{x}+\epsilon \eta_{y}\right),
\end{aligned}
$$

and so the boundary conditions are

$$
\begin{array}{rlrl}
-\eta_{x}-\epsilon \eta_{y} & =2+2 \boldsymbol{\epsilon}^{2} & & y=-1 \\
\eta_{x}-\epsilon \eta_{y} & =0 & y & =1 \\
\epsilon \eta_{x}+y \eta_{y} & =0 & x & =0,1 .
\end{array}
$$

We consider the low-friction case $\epsilon \ll 1$. Then away from the equator, where $\epsilon \ll y$, (A13) reduces to

$$
\epsilon\left(\eta_{x x}+\eta_{y y}\right)+\eta_{x}-\frac{2 \epsilon}{y} \eta_{y}=y^{2}
$$

Omitting terms involving $\epsilon$, we obtain an equation for the interior solution

$$
\eta_{x}=y^{2} .
$$

Note that this remains valid at the equator $(y=0)$, but breaks down where $y \approx \epsilon$. Boundary layers are expected at $y= \pm 1$ and $x=0$, but not at $x=1$ (see below) where the BC can thus be approximated (again dropping terms of order $\epsilon$ ) as

$$
y \eta_{y}=0 .
$$

It is readily verified that

$$
\eta(x, y)=k+(x-1) y^{2}
$$

satisfies (A20) and (A21), and thus provides an approximate solution (with error of order $\epsilon$ ) for the interior of the domain. The constant $k=1 / 6$ (in the limit $\epsilon \rightarrow$ 0 ) is determined by the requirement that the average over the domain of $\eta(x, y)$ must be zero.

The interior solution satisfies the original $\mathrm{BC}$ at $x=$ 1 , to within order $\epsilon$. To satisfy $\mathrm{BC}$ on the remaining three sides boundary layers with shorter length scales are required. Let $\tilde{\delta}$ be the boundary layer length scale at the southern (or northern) edge of the domain. For $\eta_{y y}$ to be of order one (and hence allow both the $\mathrm{BC}$ and interior solution to be matched) the nondimensional scale in the $y$ direction must satisfy $\tilde{\delta}^{-2} \epsilon=O(1)$ or $\tilde{\delta}=\epsilon^{1 / 2}$. In dimensional units $\delta=L \tilde{\delta}=L \epsilon^{1 / 2}=L\left(\kappa / f_{0}\right)^{1 / 2}=(L \kappa /$ $\beta)^{1 / 2}$. Dropping terms in $\epsilon$, and noting that the term involving $\eta_{v}$ is of order $\epsilon^{1 / 2}$, the nondimensional equation in these boundary layers is

$$
\eta_{v v}+\eta_{x}=1
$$

where $\tilde{\delta} v=y$. For the southern boundary layer, where uniform inflow is specified, one BC for the layer can be obtained from the appropriate approximation to (A16), $\eta_{x}=-2$. This implies that along the southern edge $\eta=2(1-x)+k$, where again $k \approx 1 / 6$. This shows that $\eta$ at the southern edge increases linearly to the west, as assumed in deriving the elevation scale $z_{1}$ in section $3 b$.

Considering the balance in the southern boundary layer further justifies our derivation of $z_{1}$. From (A14) and (A15) in the southern boundary layer $U=O\left(\epsilon^{-1 / 2}\right)=$ $O\left(\tilde{\delta}^{-1}\right)$ while $V=O(1)$. Thus, flow in the boundary layer is dominantly zonal, and the total mass flux is approximately independent of the boundary layer thickness (and hence the friction coefficient). This is consistent with our conclusion that essentially all of the inflowing fluid is transported zonally through this layer. Substituting these estimates for velocity scales into the nondimensional zonal momentum balance equation $(y U$ $+\epsilon V=-2 \eta_{v}$.) shows that $\epsilon V$ is negligible, and that the balance in the boundary layer is geostrophic.

Equation (A23) is identical to the one-dimensional heat conduction equation derived by Csanady (1978) for the wind forced mean circulation in a coastal zone of variable depth. Ignoring the forcing (which can be eliminated by subtracting the interior solution) in dimensional form (A23) is

$$
\eta_{y y}+(\beta H / r) \eta_{x}=0
$$


while Csanady's equation in the boundary layer at a coast with constant cross-shore slope $s=\partial_{y} H$ would be (for the $x$ axis along shore)

$$
\eta_{y v}+\left(f_{s} / r\right) \eta_{x}=0 .
$$

Csanady coined the term "arrested topographic wave" for the solution to (A25); by analogy solutions to (A24) could be called "arrested Rossby waves."

There is also a boundary layer on the west side of the domain, though this is not important to the largescale LPT elevation response. Here the nondimensional layer width is $\epsilon$, or in dimensional units $\kappa / \beta$. The nondimensional equation for this boundary layer is

$$
\eta_{x x}+\eta_{x}=0
$$

where $\epsilon \chi=x$. Equation (A26) implies that $\eta_{\chi} \propto e^{-\chi}$, showing that this boundary layer could only occur on the west side of the basin, as we noted in deriving the interior solution. Note that this is essentially the standard Stommel frictional boundary layer (e.g., Pedlosky 1987). Necessary BC for all boundary layers are readily determined from (A16)-(A18) and the interior solution (A22), and the boundary layer equations [(A23), (A26)] can be solved by standard means. These details are not important for our purposes here and will be omitted.

The interior solution is dominated by nearly geostrophically balanced flow from the western edge into the interior, with uniform divergence $\boldsymbol{\nabla} \cdot \mathbf{U}=1$ (with some outflow into the south and north boundary layers). Becuase there is no inflow on the western boundary, all of the mass required to account for the interior divergence must indeed be transported from its source along the southern edge, through the boundary layers to the western edge.

Our analysis in this appendix is strictly relevant to the low-frequency, low-friction case $\omega / f_{0} \ll \epsilon \ll 1$. In the case of Fig. 5, $\epsilon=.16$, implying a nondimensional boundary layer thickness of $\epsilon^{1 / 2}=0.4$. Thus, the north and south boundary layers, together with the zone where $\epsilon \approx y$ so that (A20) does not hold, are a substantial fraction of the model domain. The interior solution (A22) is thus not so clear in Fig. 5. When the equations are solved at low frequencies (e.g., $\omega=10^{-7} \mathrm{~s}^{-1}$ ) with a smaller friction coefficient so that $\epsilon \approx .01$, boundary layers are much smaller and the interior solution is very close to that predicted by (A22). However, if we solve the shallow-water equations with a reduced friction coefficient at the Mf frequency, the analysis of this section also fails, since in this case $\epsilon=\kappa / f_{0} \ll \omega / f_{0}$. For small $\kappa$, solutions at the Mf frequency are dominated by resonant Rossby waves, obscuring the low-frequency essentially steady geostrophic flow discussed here. We emphasize however that friction determines only the widths of the boundary layers, not the scale of the elevation change across the layer. It is this latter scale, which can be derived from considerations of geostrophy and kinematics, that determines the large-scale response to LPT forcing.

\section{REFERENCES}

Agnew, D. C., and W. R. Farrell, 1978: Self-consistent equilibrium ocean tides. Geophys. J. Roy. Astr. Soc., 55, 171-182.

Carton, J. A., 1983: The variation with frequency of the long-period tides. J. Geophys. Res., 88, 7563-7571.

Cartwright, D. E., and R. D. Ray, 1990a: Observations of the Mf ocean tide from Geosat altimetry. Geophys. Res. Lett., 17, 619-622.

phys. Res., 95, 3069-3090.

__, and - - 1991: Energetics of global ocean tides from Geosat altimetry. J. Geophys. Res., 96, 16 897-16912.

Csanady, G. T., 1978: The arrested topographic wave. J. Phys. Oceanogr., 8, 47-62.

Desai, S. D., and J. M. Wahr, 1995: Empirical ocean tide models estimated from Topex/Poseidon altimetry. J. Geophys. Res., 100, $25205-25228$.

Egbert, G. D., and S. Y. Erofeeva, 2002: Efficient inverse modeling of barotropic ocean tides. J. Atmos. Oceanic Technol., 19, 183204.

- A. F. Bennett, and M. G. G. Foreman, 1994: TOPEX/POSEIDON tides estimated using a global inverse model. J. Geophys. Res., 99, $24821-24852$.

Garrett, C., 1975: Tides in gulfs. Deep-Sea Res., 22, 23-35.

Gotlib, V. Y., B. A. Kagan, and G. A. Kivman, 1987: On the lowest gravitational mode of eigen-oscillation in the world ocean: Part 2. Sov. J. Phys, Oceanogr., 1, 11-16.

Hirose, N., I. Fukamori, and R. M. Ponte, 2001: A non-isostatic global sea level response to barometric pressure near 5 days. Geophys. Res. Lett., 28, 2441-2444.

Kagan, B. A., V. Y. Rivkind, and P. K. Chernayaev, 1976: The fortnightly lunar tides in the world ocean. Izv. Acad. Sci. USSR, Atmos. Oceanic Phys., 12, 274-276.

Longuet-Higgins, M. S., 1968: The eigenfunctions of Laplace's tidal equations over a sphere. Philos. Trans. Roy. Soc. London, 262A, $511-607$.

Luther, D. S.. 1980: Observations of long period waves in the tropical oceans and atmosphere. Ph.D. thesis, MIT and WHOI, $210 \mathrm{pp}$. 1982: Evidence for 4-6 day barotropic, planetary oscillation of the Pacific Ocean. J. Phys. Oceanogr., 12, 644-657.

Madden, R. A., and P. R. Julian, 1972: Further evidence of globalscale, 5-day pressure waves. J. Atmos. Sci., 29, 1464-1469.

Marchuk, G. I., and B. A. Kagan, 1989: Dynamics of Ocean Tides (translated from Russian). Kluwer, $327 \mathrm{pp}$.

Miller, A. J., D. S. Luther, and M. C. Hendershott, 1993: The fortnightly and monthly tides: Resonant Rossby waves or nearly equilibrium gravity waves? J. Phys. Oceanogr., 23, 879-897.

National Geophysical Data Center, 1992: GEODAS worldwide marine geophysical data. Data Announcement, 92-MGG-02, NOAA and U.S. Department of Commerce, CD-ROM.

Nerem, R. S., E. J. O. Schrama, C. K. Koblinsky, and B. D. Beckley, 1994: A preliminary evaluation of ocean topography from the Topex/Poseidon mission. J. Geophys. Res., 99, 24 565-24 583.

Pedelosky, J., 1987: Geophysical Fluid Dynamics. 2d ed. SpringerVerlag, $710 \mathrm{pp}$.

Platzman, G. W., G. A. Curtis, K. S. Hansen, and R. D. Slater, 1981: Normal modes of the world ocean. Part II: Description of modes in the period range 8 to 80 hours. J. Phys. Oceanogr., 11, 579603

Ponte, R. M., 1997: Nonequilibrium response of the global ocean to the 5-day Rossby-Haurwitz wave in atmospheric surface pressure. J. Phys. Oceanogr., 27, 2158-2168.

Proudman, J., 1960: The condition that a long-period tide shall follow the equilibrium-law. Geophys. J. Roy. Astr. Soc., 3, 244-249.

Ray, R. D., 1999: A global ocean tide model from TOPEX/POSEIDON altimetry: GOT99.2. NASA Tech Memo 209478, Goddard Space Flight Center, $58 \mathrm{pp}$.

, and D. E. Cartwright, 1994: Satellite altimeter observations of the $\mathrm{Mf}$ and $\mathrm{Mm}$ ocean tides, with simultaneous orbit corrections. Gravimetry and Space Techniques Applied to Geodynamics and 
Ocean Dynamics, B. Schutz et al., Eds., Amer. Geophys. Union, 69-78.

Shum, C. K., and Coauthors, 1997: Comparison of recent global tidal models. J. Geophys. Res., 102, 25 173-25 194.

Smith, W. H. F, and D. T. Sandwell, 1997: Global sea floor topography from satellite altimetry and ship depth soundings. Science, 277, 1956-1962.

Winant, C. D., 1979: Comments on "The arrested topographic wave." J. Phys. Oceanogr., 9, 1042-1043.
Woodworth, P. L., S. A. Windle, and J. M. Vassie, 1995: Departures from the local inverse baromter model at periods of 5 days in the central South Atlantic. J. Geophys. Res., 100, 18 281-18 290.

Wunsch, C., 1967: The long-period tides. Rev. Geophys., 5, 447-475. , and D. Stammer, 1997: Atmospheric loading and the oceanic "inverted barometer" effect. Rev. Geophys., 35, 79-107.

_- D. B. Haidvogel, M. Iskandarani, and R. Hughes, 1997: Dynamics of the long period tides. Progress in Oceanography, Vol. 40, Pergamon, 81-108. 